$\operatorname{Argonne} \underset{\bigotimes}{\bigotimes}$

\title{
September 2008 Monitoring Results for Centralia, Kansas
}

\author{
Environmental Science Division
}


About Argonne National Laboratory

Argonne is a U.S. Department of Energy laboratory managed by UChicago Argonne, LLC under contract DE-AC02-06CH11357. The Laboratory's main facility is outside Chicago, at 9700 South Cass Avenue, Argonne, Illinois 60439. For information about Argonne and its pioneering science and technology programs, see www.anl.gov.

\section{Availability of This Report}

This report is available, at no cost, at http://www.osti.gov/bridge. It is also available on paper to the U.S. Department of Energy and its contractors, for a processing fee, from:

U.S. Department of Energy

Office of Scientific and Technical Information

P.O. Box 62

Oak Ridge, TN 37831-0062

phone (865) 576-8401

fax (865) 576-5728

reports@adonis.osti.gov

\section{Disclaimer}

This report was prepared as an account of work sponsored by an agency of the United States Government. Reference herein to any specific commercial product, process, or service by trade name, trademark, manufacturer, or otherwise, does not necessarily constitute or imply its endorsement, recommendation, or favoring by the United States Government or any agency thereof. The views and opinions of document authors expressed herein do not necessarily state or reflect those of the United States Government or any agency thereof, Argonne National Laboratory, or UChicago Argonne, LLC. 


\section{September 2008 Monitoring Results for Centralia, Kansas}

by

Applied Geosciences and Environmental Management Section

Environmental Science Division, Argonne National Laboratory

February 2009

USDA

United States Department of Agriculture 


\section{Contents}

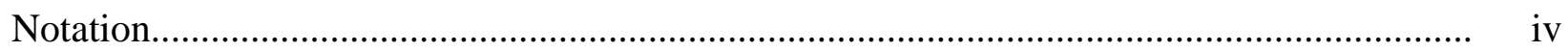

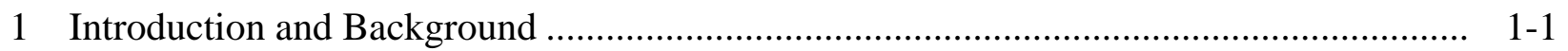

2 Sampling and Analysis Activities............................................................................ 2-1

2.1 Measurement of Groundwater Levels..................................................................... 2-1

2.2 Monitoring Well and Piezometer Sampling and Analyses ........................................ 2-1

2.3 Handling and Disposal of Investigation-Derived Waste .......................................... 2-2

2.4 Quality Control for Sample Collection, Handling, and Analysis ............................. 2-2

3 Results and Discussion ..................................................................................... 3-1

3.1 Groundwater Level Data....................................................................... 3-1

3.2 Groundwater Analysis Results.......................................................................... 3-1

3.2.1 Results of the Groundwater Analyses for VOCs ........................................... 3-1

3.2.2 Results of Other Groundwater Analyses.......................................................... 3-2

4 Conclusions and Recommendations …………............................................................ 4-1

4.1 Conclusions .................................................................................................. 4-1

4.2 Recommendations...................................................................................... 4-2

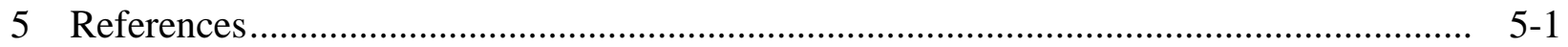

Appendix A: Sequence of Sampling Activities at Centralia, Kansas, in September 2008..... A-1

Appendix B: Data Summary for Verification VOCs Analyses by TestAmerica Laboratories, Inc.

\section{Figures}

$1.1 \quad$ Approved monitoring network at Centralia .............................................................. 1-3

1.2 Locations of pilot test injection points and post-injection groundwater monitoring points PMP1-PMP9

3.1 Potentiometric surface at Centralia, based on water levels measured manually on June 25, 2008

3.2 Carbon tetrachloride levels in groundwater at Centralia in September 2008, with the interpreted lateral extent of the contaminant at intervals during the period August 2004 to September 2008

4.1 Previously established monitoring points proposed for ongoing annual monitoring ... 4-4

4.2 Pilot test monitoring points proposed for ongoing annual monitoring or twice yearly monitoring..... 


\section{Tables}

3.1 Hand-measured water levels at Centralia in November 2007, March 2008, June 2008, and September 2008

3.2 Analytical results from the AGEM Laboratory for volatile organic compounds in groundwater samples collected at Centralia, August 2004 to September 2008.

3.3 Field measurements for groundwater samples collected at Centralia, August 2004 to September 2008 


\section{Notation}

AGEM Applied Geosciences and Environmental Management

AMSL above mean sea level

BGL below ground level

${ }^{\circ} \mathrm{C} \quad$ degree(s) Celsius

CCC Commodity Credit Corporation

COC chain of custody

EPA U.S. Environmental Protection Agency

$\mathrm{ft} \quad$ foot (feet)

in. inch(es)

ISCR in situ chemical reduction

KDHE Kansas Department of Health and Environment

L liter(s)

$\mu g / L \quad$ microgram(s) per liter

$\mu \mathrm{S} / \mathrm{cm} \quad$ microsiemen(s) per centimeter

mg/L milligram(s) per liter

$\mathrm{mV} \quad$ millivolt(s)

NAD North American Datum

NGVD National Geodetic Vertical Datum

ORP oxidation-reduction potential

TOC top of casing

USDA U.S. Department of Agriculture

VOC volatile organic compound 


\section{September 2008 Monitoring Results for Centralia, Kansas}

\section{Introduction and Background}

In September 2005, periodic sampling of groundwater was initiated by the Commodity Credit Corporation of the U.S. Department of Agriculture (CCC/USDA) in the vicinity of a grain storage facility formerly operated by the CCC/USDA at Centralia, Kansas. The sampling at Centralia is being performed on behalf of the CCC/USDA by Argonne National Laboratory, in accord with a monitoring program approved by the Kansas Department of Health and Environment (KDHE). The objective is to monitor levels of carbon tetrachloride contamination identified in the groundwater at Centralia (Argonne 2003, 2004, 2005a). Under a KDHEapproved monitoring plan (Argonne 2005b), the groundwater was sampled twice yearly (for a recommended period of two years) for analyses for volatile organic compounds (VOCs), as well as measurement of selected geochemical parameters to aid in the evaluation of possible natural contaminant degradation (reductive dechlorination) processes in the subsurface environment.

The sampling in September 2007 represented the fifth and final monitoring event performed under the two-year twice yearly monitoring program (Argonne 2006a,b, 2007a, 2008a). The results from the two-year monitoring program demonstrated the presence of carbon tetrachloride contamination at levels exceeding the KDHE Tier 2 Risk-Based Screening Level of $5 \mu \mathrm{g} / \mathrm{L}$ for this compound in a broad groundwater plume that has shown little movement. The relative concentrations of chloroform, the primary degradation product of carbon tetrachloride, suggested that some degree of reductive dechlorination or natural biodegradation was taking place in situ at the former CCC/USDA facility on a localized scale.

The CCC/USDA subsequently developed an Interim Measure Conceptual Design (Argonne 2007b), proposing a pilot test of the Adventus EHC in situ chemical reduction technology, that was approved by the KDHE in November 2007 (KDHE 2007). Implementation of the proposed interim measure occurred in December 2007. The objective was to create highly reducing conditions that would enhance both chemical and biological reductive dechlorination in the injection test area (Argonne 2009).

The KDHE has requested that sitewide monitoring continue at Centralia until a final remedy has been implemented and a comprehensive program of performance and compliance monitoring has been established for this site (KDHE 2008a). The groundwater sampling events 
at Centralia in March 2008 (Argonne 2008b) and September 2008 were performed in response to this request. The present report documents the results of groundwater sampling conducted in September 2008, in the network of 10 monitoring wells and 6 piezometers (Figure 1.1) previously approved by the KDHE for extended monitoring of the groundwater contamination at Centralia (KDHE 2005a,b). Sampling of additional piezometers specifically installed to monitor the progress of the interim measure pilot test (PMP1-PMP9; Figure 1.2) was also performed in September 2008; the results of these analyses are reported and discussed separately (Argonne 2009). 


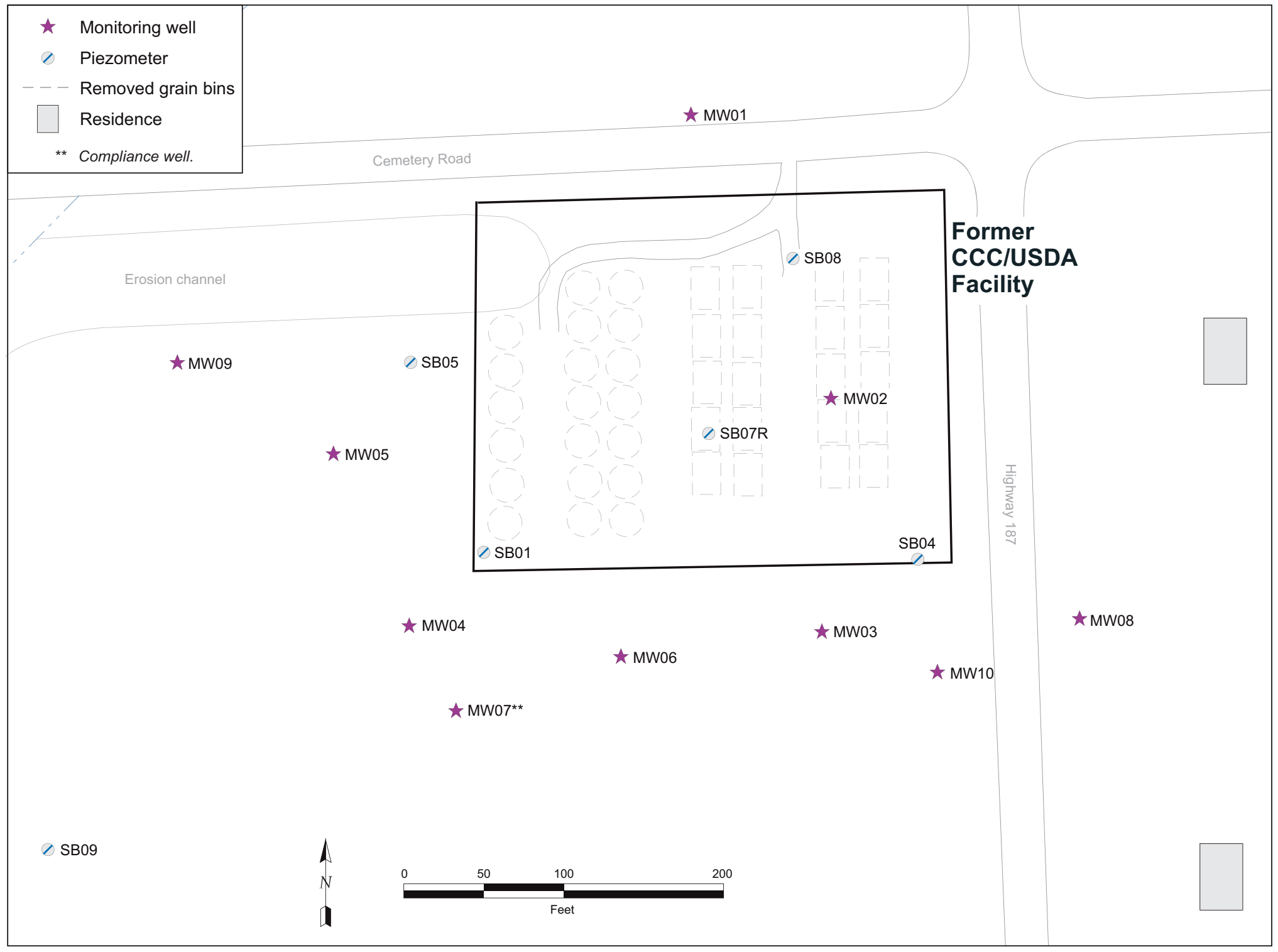

FIGURE 1.1 Approved monitoring network at Centralia. 


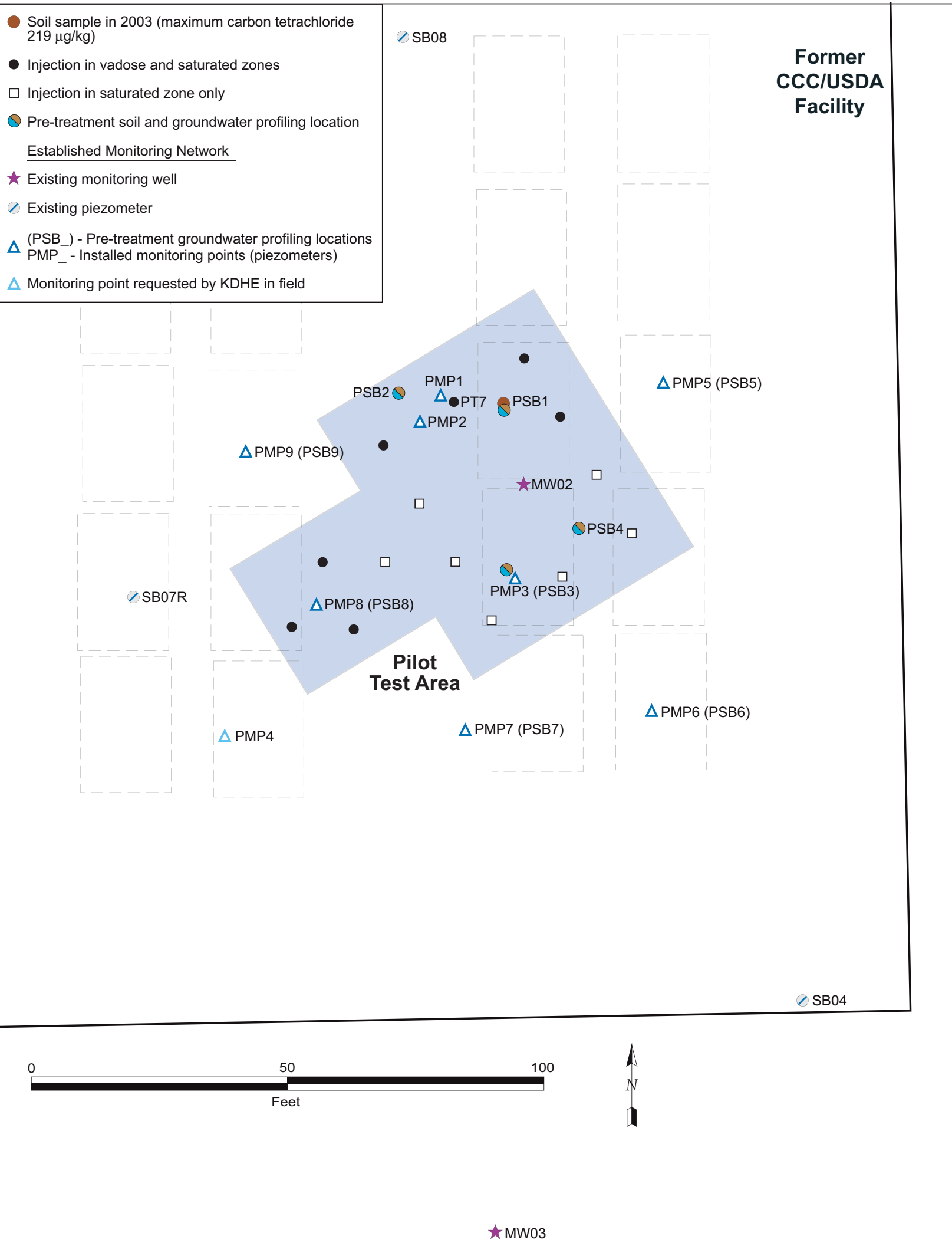

FIGURE 1.2 Locations of pilot test injection points and post-injection groundwater monitoring points PMP1-PMP9. 


\section{Sampling and Analysis Activities}

\subsection{Measurement of Groundwater Levels}

Monitoring wells MW01-MW10 and piezometers SB01, SB04, SB05, SB07R, SB08, and SB09 (Figure 1.1) were sampled on September 8-10, 2008. Before the sampling, a water level indicator was used to measure the depth to groundwater and the total depth of each well, to within $0.01 \mathrm{ft}$, from the top of the well casing. Samples were collected by using a low-flow bladder pump and a Waterra pump, with the approval of the KDHE (2008b).

Data recorders currently installed in MW01-MW06 are gathering long-term data on the groundwater elevation and gradient at Centralia. The data recorders in these wells were downloaded, and water levels were measured manually at all monitoring points, on June 25, 2008.

The groundwater level data are discussed in Section 3.1.

\subsection{Monitoring Well and Piezometer Sampling and Analyses}

After measurement of water levels, each monitoring point was purged of a small volume by using low-flow techniques, in accord with U.S. Environmental Protection Agency (EPA) procedure EPA/540/S-95/504 (Puls and Barcelona 1996) and the equipment manufacturers' instructions. Field measurements of temperature, $\mathrm{pH}$, conductivity, dissolved oxygen (DO), and oxidation reduction potential (ORP) were taken during purging until the measurements stabilized. Field measurements of iron(II) were made as outlined in the monitoring plan (Argonne 2005b), in accord with procedures in the Master Work Plan (Argonne 2002). The sequence of activities during the September 2008 well sampling event is summarized in Appendix A.

Groundwater samples designated for VOCs analyses and selected geochemical analyses identified in the monitoring plan (Argonne 2005b) were collected in appropriate laboratory containers, labeled, packaged, and chilled to $4^{\circ} \mathrm{C}$ by placement in ice-filled coolers. The samples were shipped by an overnight delivery service to the Applied Geosciences and Environmental Management (AGEM) Laboratory at Argonne for VOCs analyses with EPA Method 524.2 (EPA 
1995). Aliquots of selected samples (chosen in the field) were also shipped to TestAmerica Laboratories, Inc., South Burlington, Vermont, for verification VOCs analyses.

The analytical results for groundwater samples are discussed in Section 3.2.

\subsection{Handling and Disposal of Investigation-Derived Waste}

The small volume of purge water generated as potentially contaminated investigationderived waste was containerized on-site. After sampling and analysis by a KDHE-certified laboratory (see Section 2.4), the accumulated purge water was taken to the Sabetha, Kansas, publicly owned treatment works on December 11, 2008, for disposal, per verbal approval of the KDHE.

\subsection{Quality Control for Sample Collection, Handling, and Analysis}

Quality assurance/quality control procedures followed during the September 2008 monitoring event are described in detail in the Master Work Plan (Argonne 2002). The results are summarized as follows:

- Sample collection and handling activities were monitored by the documentation of samples as they were collected and the use of chain-ofcustody forms and custody seals to ensure sample integrity during handling and shipment.

- Samples designated for VOCs analyses were received with custody seals intact and at the appropriate preservation temperature. All samples were analyzed within the required holding times.

- Quality control samples collected to monitor sample collection and handling activities included equipment rinsates and trip blanks. In addition, method blanks were analyzed with the samples to monitor analytical methodologies. All quality control samples analyzed at the AGEM Laboratory were free of carbon tetrachloride and chloroform contamination. 
- Groundwater samples were analyzed for VOCs at the AGEM Laboratory with the purge-and-trap method on a gas chromatograph-mass spectrometer system. Calibration checks with each sample delivery group were required to be within $\pm 20 \%$ of the standard. Surrogate standard determinations performed on samples and blanks were within the specified range of $80-120 \%$ for all samples, in either the initial analysis or a successful reanalysis.

- In accordance with the quality control procedures defined in the Master Work Plan (Argonne 2002), the analyses of water samples at the AGEM Laboratory were verified by a second laboratory. Three groundwater samples collected during the September 2008 monitoring event (from monitoring wells MW01, MW04, and PMP1) were submitted for verification organic analysis according to EPA Contract Laboratory Program methodology by TestAmerica. Results show good agreement over the range of contaminant concentrations detected, with average relative percent difference values of $7.7 \%$ for carbon tetrachloride and $7.4 \%$ for chloroform. Summary pages for the verification organic analyses by TestAmerica for samples collected on September 8-10, 2008, are in Appendix B. 


\section{Results and Discussion}

\subsection{Groundwater Level Data}

Depths to groundwater were measured manually in all available monitoring wells on November 12, 2007, March 12-20, 2008 (during sampling), June 25, 2008, and September 9-10, 2008 (during sampling). The hand-measured water level data are in Table 3.1.

The potentiometric surface at Centralia, interpreted from manual measurements on June 25, 2008, is depicted in Figure 3.1. The recent results are consistent with previous measurements (Argonne 2006a, 2007a, 2008a,b), indicating an apparent groundwater flow direction toward the southwest across much of the former CCC/USDA facility. Like previous depictions of the potentiometric surface, Figure 3.1 indicates that groundwater flow is focused toward a localized low in the potentiometric surface, defined by the water level measurements at SB01, MW04, MW06, and MW07. Argonne's earlier investigations (Argonne 2003, 2004) suggested that the increased hydraulic gradients observed near these wells are a reflection of relatively lowpermeability silts and clays that comprise the aquifer unit in this portion of the study area, in comparison to the more coarse-grained deposits identified in the northern and eastern portions of the site. The results of groundwater analyses at Centralia (discussed in Section 3.2) support an interpretation of slow groundwater flow (and carbon tetrachloride migration) to the southsouthwest, in keeping with the observed water level patterns.

\subsection{Groundwater Analysis Results}

\subsubsection{Results of the Groundwater Analyses for VOCs}

The analytical data for VOCs in the groundwater samples collected in September 2008 are in Table 3.2, together with data for the previous sampling events conducted at Centralia since sampling of the monitoring well network began in 2004. The September 2008 data for carbon tetrachloride are illustrated in Figure 3.2, along with the lateral margins of the plume, as interpreted by Argonne on the basis of each of the groundwater sampling events summarized in Table 3.2. 
Carbon tetrachloride was detected in September 2008 at 10 of the 16 approved monitoring locations (KDHE 2005a,b) on and downgradient from the former CCC/USDA facility (Figure 3.2), at concentrations ranging from $2.0 \mu \mathrm{g} / \mathrm{L}$ (at MW04) to a maximum of $378 \mu \mathrm{g} / \mathrm{L}$ (at SB01). Chloroform concentrations ranging from $<1 \mu \mathrm{g} / \mathrm{L}$ to $57 \mu \mathrm{g} / \mathrm{L}$ were detected at 9 of the 16 sampled locations (Table 3.2). The decrease in carbon tetrachloride concentration noted at MW02 (from 1,138 $\mu \mathrm{g} / \mathrm{L}$ in September 2007 to no detection in March 2008 to $18 \mu \mathrm{g} / \mathrm{L}$ in September 2008) and the corresponding increase in chloroform and methylene chloride at that location are related to the interim measure pilot test (Argonne 2007b) initiated in December 2007. The results of the pilot test were reported separately (Argonne 2009).

Except for the noted change at MW02, the present carbon tetrachloride concentrations in sitewide monitoring wells are consistent with previous measurements. The data in Table 3.2 and Figure 3.2 continue to suggest longer-term trends of slightly increasing carbon tetrachloride levels at monitoring points SB05, MW03, MW04, and MW07, along the western and southern margins of the groundwater plume and in the apparent direction of groundwater flow.

\subsubsection{Results of Other Groundwater Analyses}

The results of field measurements on the groundwater samples are summarized in Table 3.3. Additional parameters were formerly reported to estimate whether the in situ conditions at Centralia are suitable for possible degradation of carbon tetrachloride by natural anaerobic (reductive dechlorination) processes, as outlined in regulatory guidance for the evaluation of these conditions (KDHE 2001; EPA 1998). Because the results gave only limited evidence for possible anaerobic biodegradation of carbon tetrachloride at Centralia, the analyses for additional parameters have been discontinued. The persistent occurrence of the carbon tetrachloride breakdown product chloroform at relatively elevated concentrations at monitoring points SB01, SB05, and MW02 during the March 2005 sampling event and subsequently (Table 3.2) suggested that carbon tetrachloride degradation was occurring at these locations. 
TABLE 3.1 Hand-measured water levels at Centralia in November 2007, March 2008, June 2008, and September 2008.

\begin{tabular}{|c|c|c|c|c|c|c|c|c|c|}
\hline \multirow[b]{2}{*}{ Well } & \multirow[b]{2}{*}{$\begin{array}{l}\text { Top of Casing } \\
\text { Elevation } \\
\text { (ft AMSL) }\end{array}$} & \multicolumn{2}{|c|}{ November 12, 2007} & \multicolumn{2}{|c|}{ March 12-20, $2008^{a}$} & \multicolumn{2}{|c|}{ June 25, 2008} & \multicolumn{2}{|c|}{ September 8-10, $2008^{a}$} \\
\hline & & $\begin{array}{l}\text { Depth to } \\
\text { Groundwater } \\
\text { (ft TOC) }\end{array}$ & $\begin{array}{c}\text { Groundwater } \\
\text { Elevation } \\
\text { (ft AMSL) }\end{array}$ & $\begin{array}{l}\text { Depth to } \\
\text { Groundwater } \\
\text { (ft TOC) }\end{array}$ & $\begin{array}{c}\text { Groundwater } \\
\text { Elevation } \\
\text { (ft AMSL) }\end{array}$ & $\begin{array}{l}\text { Depth to } \\
\text { Groundwaterb } \\
\text { (ft TOC) }\end{array}$ & $\begin{array}{l}\text { Groundwater } \\
\text { Elevation } \\
\text { (ft AMSL) }\end{array}$ & $\begin{array}{l}\text { Depth to } \\
\text { Groundwaterb } \\
\text { (ft TOC) }\end{array}$ & $\begin{array}{c}\text { Groundwater } \\
\text { Elevation } \\
\text { (ft AMSL) }\end{array}$ \\
\hline MW01 & 1329.30 & 13.95 & 1315.35 & 11.89 & 1317.41 & 9.88 & 1319.42 & 13.6 & 1315.70 \\
\hline MW02 & 1334.82 & 22.68 & 1312.14 & 21.85 & 1312.97 & 20.25 & 1314.57 & 21.9 & 1312.92 \\
\hline MW03 & 1334.70 & 22.54 & 1312.16 & 21.22 & 1313.48 & 19.65 & 1315.05 & 20.8 & 1313.90 \\
\hline MW04 & 1322.71 & 26.06 & 1296.65 & 24.23 & 1298.48 & 23.10 & 1299.61 & 24.0 & 1298.71 \\
\hline MW05 & 1318.11 & 10.49 & 1307.62 & 7.32 & 1310.79 & 8.36 & 1309.75 & 10.0 & 1308.11 \\
\hline MW06 & 1329.82 & 38.47 & 1291.35 & 34.70 & 1295.12 & 36.50 & 1293.32 & 36.6 & 1293.22 \\
\hline MW07 & 1324.83 & 29.59 & 1295.24 & 28.32 & 1296.51 & 26.60 & 1298.23 & 28.2 & 1296.63 \\
\hline MW08 & 1332.41 & 20.40 & 1312.01 & 18.85 & 1313.56 & 17.80 & 1314.61 & 18.6 & 1313.81 \\
\hline MW09 & 1310.49 & 2.89 & 1307.60 & 0.00 & 1310.49 & 0.61 & 1309.88 & 2.5 & 1307.99 \\
\hline MW10 & 1334.56 & 22.36 & 1312.20 & 20.90 & 1313.66 & 19.52 & 1315.04 & 21.1 & 1313.46 \\
\hline SB01 & 1325.16 & 19.72 & 1305.44 & 15.87 & 1309.29 & 16.23 & 1308.93 & 20.1 & 1305.06 \\
\hline SBO4 & 1335.73 & 23.68 & 1312.05 & 22.34 & 1313.39 & 20.69 & 1315.04 & 21.9 & 1313.83 \\
\hline SB05 & 1321.28 & - & - & 7.43 & 1313.85 & 7.50 & 1313.78 & 10.6 & 1310.68 \\
\hline SB07R & 1331.71 & 19.56 & 1312.15 & 18.23 & 1313.48 & 16.63 & 1315.08 & 18.4 & 1313.31 \\
\hline SB08 & 1332.56 & 20.49 & 1312.07 & 19.24 & 1313.32 & 17.41 & 1315.15 & 18.6 & 1313.96 \\
\hline SB09 & 1311.04 & 6.27 & 1304.77 & 2.90 & 1308.14 & 6.47 & 1304.57 & 6.6 & 1304.44 \\
\hline
\end{tabular}

a Measurements made during sampling.

b Depths measured from the top of the casing (TOC). 
TABLE 3.2 Analytical results from the AGEM Laboratory for volatile organic compounds in groundwater samples collected at Centralia, August 2004 to September 2008.

\begin{tabular}{|c|c|c|c|c|c|c|}
\hline \multirow[b]{2}{*}{ Well } & \multirow{2}{*}{$\begin{array}{l}\text { Screen } \\
\text { Interval } \\
\text { (ft BGL) }\end{array}$} & \multirow[b]{2}{*}{ Sample } & \multirow[b]{2}{*}{$\begin{array}{c}\text { Sampling } \\
\text { Date }\end{array}$} & \multicolumn{3}{|c|}{ Concentration $(\mu \mathrm{g} / \mathrm{L})$} \\
\hline & & & & $\begin{array}{c}\text { Carbon } \\
\text { Tetrachloride }\end{array}$ & Chloroform & $\begin{array}{c}\text { Methylene } \\
\text { Chloride }\end{array}$ \\
\hline
\end{tabular}

Wells in the approved sitewide monitoring network

\begin{tabular}{|c|c|c|c|c|c|c|}
\hline \multirow[t]{10}{*}{ MW01 } & \multirow[t]{10}{*}{$54.5-64.5$} & CNMW01-W-16158 & $8 / 24 / 04$ & $N D^{a}$ & ND & ND \\
\hline & & CNMW01-W-19276 & 9/10/05 & ND & ND & ND \\
\hline & & CNMW01-W-16308 & $10 / 11 / 05$ & ND & ND & ND \\
\hline & & CNMW01-W-19890 & $3 / 15 / 06$ & ND & ND & ND \\
\hline & & CNMW01-W-22501 & $9 / 25 / 06$ & ND & ND & ND \\
\hline & & CNMW01-W-16326 & $3 / 29 / 07$ & ND & ND & ND \\
\hline & & CNMW01-W-16228 & 9/26/07 & $1 \mathrm{R}^{\mathrm{b}}$ & ND & ND \\
\hline & & CNMW01-W-26082 & $1 / 11 / 08$ & ND & ND & ND \\
\hline & & CNMW01-W-26023 & $3 / 19 / 08$ & ND & ND & ND \\
\hline & & CNMW01-W-26673 & 9/9/08 & ND & ND & ND \\
\hline \multirow[t]{9}{*}{ MW02 } & \multirow[t]{9}{*}{$49.5-59.5$} & CNMW02-W-16159 & $8 / 26 / 04$ & 215 & 6.2 & ND \\
\hline & & CNMW02-W-19282 & 9/11/05 & 776 & 33 & ND \\
\hline & & CNMW02-W-16309 & $10 / 12 / 05$ & 528 & 21 & ND \\
\hline & & CNMW02-W-19908 & $3 / 16 / 06$ & 847 & 21 & ND \\
\hline & & CNMW02-W-22508 & $9 / 26 / 06$ & 1233 & 25 & ND \\
\hline & & CNMW02-W-15489 & $3 / 26 / 07$ & 829 & 14 & ND \\
\hline & & CNMW02-W-16227 & $9 / 26 / 07$ & 1138 & 18 & ND \\
\hline & & CNMW02-W-26000 & $3 / 12 / 08$ & ND & 1.2 & 1.9 \\
\hline & & CNMW02-W-26674 & $9 / 8 / 08$ & 18 & 57 & 11 \\
\hline \multirow[t]{9}{*}{ MW03 } & \multirow[t]{9}{*}{$50.5-60.5$} & CNMW03-W-16178 & $8 / 24 / 04$ & 1.2 & ND & ND \\
\hline & & CNMW03-W-19277 & 9/10/05 & 1.6 & ND & ND \\
\hline & & CNMW03-W-16310 & $10 / 11 / 05$ & 1.8 & ND & ND \\
\hline & & CNMW03-W-19909 & $3 / 17 / 06$ & 2.6 & $0.2 \mathrm{Jc}^{\mathrm{c}}$ & ND \\
\hline & & CNMW03-W-22513 & 9/26/06 & 2.7 & ND & ND \\
\hline & & CNMW03-W-15494 & $3 / 27 / 07$ & 2.5 & ND & ND \\
\hline & & CNMW03-W-16223 & $9 / 25 / 07$ & 3.5 & ND & ND \\
\hline & & CNMW03-W-26001 & $3 / 12 / 08$ & 2.3 & ND & ND \\
\hline & & CNMW03-W-26675 & 9/9/08 & 3.2 & $0.3 \mathrm{~J}$ & ND \\
\hline \multirow[t]{9}{*}{ MW04 } & \multirow[t]{9}{*}{$37.5-47.5$} & CNMW04-W-16180 & $8 / 24 / 04$ & ND & ND & ND \\
\hline & & CNMW04-W-19280 & $9 / 11 / 05$ & $0.9 \mathrm{~J}$ & ND & ND \\
\hline & & CNMW04-W-16311 & $10 / 11 / 05$ & $0.8 \mathrm{~J}$ & ND & ND \\
\hline & & CNMW04-W-19891 & $3 / 15 / 06$ & 1.3 & ND & ND \\
\hline & & CNMW04-W-22506 & $9 / 25 / 06$ & 1.4 & $0.1 \mathrm{~J}$ & ND \\
\hline & & CNMW04-W-16210 & $3 / 28 / 07$ & 2.1 & ND & ND \\
\hline & & CNMW04-W-16220 & $9 / 24 / 07$ & 2.0 & ND & ND \\
\hline & & CNMW04-W-26024 & 3/19/08 & 1.3 & ND & ND \\
\hline & & CNMW04-W-26676 & 9/9/08 & 2.0 & ND & ND \\
\hline \multirow[t]{9}{*}{ MW05 } & \multirow[t]{9}{*}{$34.5-44.5$} & CNMW05-W-16183 & $8 / 25 / 04$ & ND & ND & ND \\
\hline & & CNMW05-W-19279 & 9/10/05 & 1.9 & ND & ND \\
\hline & & CNMW05-W-16312 & $10 / 11 / 05$ & 1.5 & ND & ND \\
\hline & & CNMW05-W-19976 & $3 / 15 / 06$ & 1.3 & ND & ND \\
\hline & & CNMW05-W-22505 & $9 / 25 / 06$ & 1.3 & ND & ND \\
\hline & & CNMW05-W-16213 & $3 / 28 / 07$ & $0.5 \mathrm{~J}$ & ND & ND \\
\hline & & CNMW05-W-16218 & $9 / 24 / 07$ & 1.2 & ND & ND \\
\hline & & CNMW05-W-26025 & 3/19/08 & 1.9 & ND & ND \\
\hline & & CNMW05-W-26677 & 9/10/08 & 13 & $0.7 \mathrm{~J}$ & ND \\
\hline
\end{tabular}


TABLE 3.2 (Cont.)

\begin{tabular}{|c|c|c|c|c|c|c|}
\hline \multirow[b]{2}{*}{ Well } & \multirow[b]{2}{*}{$\begin{array}{l}\text { Screen } \\
\text { Interval } \\
\text { (ft BGL) }\end{array}$} & \multirow[b]{2}{*}{ Sample } & \multirow[b]{2}{*}{$\begin{array}{l}\text { Sampling } \\
\text { Date }\end{array}$} & \multicolumn{3}{|c|}{ Concentration $(\mu \mathrm{g} / \mathrm{L})$} \\
\hline & & & & $\begin{array}{c}\text { Carbon } \\
\text { Tetrachloride }\end{array}$ & Chloroform & $\begin{array}{c}\text { Methylene } \\
\text { Chloride }\end{array}$ \\
\hline
\end{tabular}

Wells in the approved sitewide monitoring network (cont.)

\begin{tabular}{|c|c|c|c|c|c|c|}
\hline MW06 & $46.5-56.5$ & CNMW06-W-16184 & $8 / 25 / 04$ & ND & ND & ND \\
\hline & & CNMW06-W-19278 & 9/10/05 & ND & ND & ND \\
\hline & & CNMW06-W-16313 & 10/11/05 & $0.3 \mathrm{~J}$ & ND & ND \\
\hline & & CNMW06-W-19889 & $3 / 15 / 06$ & $0.2 \mathrm{~J}$ & ND & ND \\
\hline & & CNMW06-W-22511 & $9 / 27 / 06$ & ND & ND & ND \\
\hline & & CNMW06-W-16208 & $3 / 27 / 07$ & ND & ND & ND \\
\hline & & CNMW06-W-16222 & 9/24/07 & ND & ND & ND \\
\hline & & CNMW06-W-26026 & 3/19/08 & ND & ND & ND \\
\hline & & CNMW06-W-26678 & 9/9/08 & ND & ND & ND \\
\hline MW07 & $45-55$ & CNMW07-W-19887 & $3 / 14 / 06$ & $0.4 \mathrm{~J}$ & $0.6 \mathrm{~J}$ & ND \\
\hline & & CNMW07-W-22512 & 9/26/06 & 1.1 & ND & ND \\
\hline & & CNMW07-W-15492 & 3/26/07 & 1.8 & ND & ND \\
\hline & & CNMW07-W-16221 & 9/24/07 & 2.4 & ND & ND \\
\hline & & CNMW07-W-26027 & $3 / 19 / 08$ & 3.0 & ND & ND \\
\hline & & CNMW07-W-26679 & 9/9/08 & 4.0 & $0.2 \mathrm{~J}$ & ND \\
\hline MW08 & $38-53$ & CNMW08-W-19284 & $3 / 14 / 06$ & ND & ND & ND \\
\hline & & CNMW08-W-22507 & $9 / 26 / 06$ & ND & ND & ND \\
\hline & & CNMW08-W-15493 & $3 / 27 / 07$ & ND & ND & ND \\
\hline & & CNMW08-W-16226 & $9 / 25 / 07$ & ND & ND & ND \\
\hline & & CNMW08-W-26028 & $3 / 20 / 08$ & ND & ND & ND \\
\hline & & CNMW08-W-26680 & 9/10/08 & ND & ND & ND \\
\hline MW09 & $25-35$ & CNMW09-W-19285 & $3 / 15 / 06$ & ND & ND & ND \\
\hline & & CNMW09-W-22504 & $9 / 25 / 06$ & ND & ND & ND \\
\hline & & CNMW09-W-16209 & $3 / 27 / 07$ & ND & ND & ND \\
\hline & & CNMW09-W-16219 & 9/24/07 & ND & ND & ND \\
\hline & & CNMW09-W-26029 & $3 / 20 / 08$ & ND & ND & ND \\
\hline & & CNMW09-W-26681 & 9/10/08 & ND & ND & ND \\
\hline MW10 & $30-45$ & CNMW10-W-19886 & $3 / 14 / 06$ & ND & ND & ND \\
\hline & & CNMW10-W-22510 & 9/26/06 & ND & ND & ND \\
\hline & & CNMW10-W-16215 & $3 / 28 / 07$ & ND & ND & ND \\
\hline & & CNMW10-W-16224 & $9 / 25 / 07$ & ND & ND & ND \\
\hline & & CNMW10-W-26030 & $3 / 20 / 08$ & ND & ND & ND \\
\hline & & CNMW10-W-26682 & 9/9/08 & ND & ND & ND \\
\hline SB01 & $40-50$ & CNSB01-W-16188 & $8 / 26 / 04$ & 186 & 6.5 & ND \\
\hline & & CNSB01-W-19274 & 9/9/05 & 269 & 6.8 & ND \\
\hline & & CNSB01-W-16314 & $10 / 12 / 05$ & 288 & 6.6 & ND \\
\hline & & CNSB01-W-19979 & 3/17/06 & 320 & 5.7 & ND \\
\hline & & CNSB01-W-22516 & 9/27/06 & 267 & 6.3 & ND \\
\hline & & CNSB01-W-15491 & $3 / 27 / 07$ & 222 & 4.9 & ND \\
\hline & & CNSB01-W-16232 & $9 / 27 / 07$ & 283 & 4.6 & ND \\
\hline & & CNSB01-W-26031 & $3 / 20 / 08$ & 325 & 4.8 & ND \\
\hline & & CNSB01-W-26683 & 9/10/08 & 378 & 4.1 & ND \\
\hline
\end{tabular}


TABLE 3.2 (Cont.)

\begin{tabular}{|c|c|c|c|c|c|c|}
\hline \multirow[b]{2}{*}{ Well } & \multirow[b]{2}{*}{$\begin{array}{l}\text { Screen } \\
\text { Interval } \\
\text { (ft BGL) }\end{array}$} & \multirow[b]{2}{*}{ Sample } & \multirow[b]{2}{*}{$\begin{array}{l}\text { Sampling } \\
\text { Date }\end{array}$} & \multicolumn{3}{|c|}{ Concentration $(\mu \mathrm{g} / \mathrm{L})$} \\
\hline & & & & $\begin{array}{c}\text { Carbon } \\
\text { Tetrachloride }\end{array}$ & Chloroform & $\begin{array}{c}\text { Methylene } \\
\text { Chloride }\end{array}$ \\
\hline
\end{tabular}

Wells in the approved sitewide monitoring network (cont.)

\begin{tabular}{|c|c|c|c|c|c|c|}
\hline \multirow[t]{7}{*}{ SB04 } & $51-61$ & $\begin{array}{l}\text { CNSB04-W-16189 } \\
\text { CNSB04-W-19273 } \\
\text { CNSB04-W-16315 }\end{array}$ & $\begin{array}{r}8 / 26 / 04 \\
9 / 9 / 05 \\
10 / 12 / 05\end{array}$ & $\begin{array}{l}30 \\
47 \\
44\end{array}$ & $\begin{array}{l}\text { ND } \\
\quad 0.6 \mathrm{~J} \\
0.5 \mathrm{~J}\end{array}$ & $\begin{array}{l}\text { ND } \\
\text { ND } \\
\text { ND }\end{array}$ \\
\hline & & CNSB04-W-19906 & 3/16/06 & 51 & $0.5 \mathrm{~J}$ & $0.4 \mathrm{~J} \mathrm{~B}^{\mathrm{d}}$ \\
\hline & & CNSB04-W-22503 & 9/25/06 & 54 & $0.7 \mathrm{~J}$ & ND \\
\hline & & CNSB04-W-16216 & $3 / 28 / 07$ & 44 & $0.5 \mathrm{~J}$ & ND \\
\hline & & CNSB04-W-16230 & $9 / 26 / 07$ & 36 & $0.4 \mathrm{~J}$ & ND \\
\hline & & CNSB04-W-26002 & 3/12/08 & 30 & $0.3 \mathrm{~J}$ & ND \\
\hline & & CNSB04-W-26684 & 9/9/08 & 15 & $0.3 \mathrm{~J}$ & ND \\
\hline \multirow[t]{9}{*}{ SB05 } & $32-42$ & CNSB05-W-16190 & 8/26/04 & 59 & 5.5 & ND \\
\hline & & CNSB05-W-19275 & 9/9/05 & 77 & 7.2 & ND \\
\hline & & CNSB05-W-16323 & $10 / 12 / 05$ & 54 & 5.5 & ND \\
\hline & & CNSB05-W-19904 & 3/17/06 & 104 & 7.2 & ND \\
\hline & & CNSB05-W-19940 & 9/27/06 & 139 & 12 & ND \\
\hline & & CNSB05-W-16212 & $3 / 28 / 07$ & 138 & 12 & ND \\
\hline & & CNSB05-W-16233 & $9 / 26 / 07$ & 221 & 16 & ND \\
\hline & & CNSB05-W-26032 & 3/20/08 & 224 & 17 & ND \\
\hline & & CNSB05-W-26685 & 9/9/08 & 256 & 20 & ND \\
\hline \multirow[t]{6}{*}{ SB07R } & $45-60$ & CNSB07R-W-19978 & $3 / 15 / 06$ & 41 & 2.7 & ND \\
\hline & & CNSB07R-W-19924 & $9 / 26 / 06$ & 30 & 1.7 & ND \\
\hline & & CNSB07R-W-15490 & $3 / 26 / 07$ & 30 & 1.7 & ND \\
\hline & & CNSB07R-W-16225 & $9 / 25 / 07$ & 50 & 2.4 & ND \\
\hline & & CNSB07R-W-26003 & $3 / 12 / 08$ & 13 & $0.9 \mathrm{~J}$ & ND \\
\hline & & CNSB07R-W-26686 & 9/9/08 & 21 & $1.4 \mathrm{~J}$ & ND \\
\hline \multirow[t]{9}{*}{ SB08 } & $52-62$ & CNSB08-W-16192 & 8/26/04 & 79 & 3.1 & ND \\
\hline & & CNSB08-W-19272 & 9/8/05 & 80 & 2.6 & ND \\
\hline & & CNSB08-W-16317 & $10 / 12 / 05$ & 77 & 2.8 & ND \\
\hline & & CNSB08-W-19903 & $3 / 17 / 06$ & 91 & 2.7 & ND \\
\hline & & CNSB08-W-22500 & 9/21/06 & 53 & 1.6 & ND \\
\hline & & CNSB08-W-16214 & $3 / 28 / 07$ & 64 & 2.0 & ND \\
\hline & & CNSB08-W-16229 & $9 / 26 / 07$ & 68 & 1.8 & ND \\
\hline & & CNSB08-W-26004 & 3/12/08 & 28 & 1.1 & ND \\
\hline & & CNSB08-W-26687 & 9/8/08 & 22 & $1.2 \mathrm{~J}$ & ND \\
\hline \multirow[t]{9}{*}{ SB09 } & $32-42$ & CNSB09-W-16193 & 8/26/04 & ND & ND & ND \\
\hline & & CNSB09-W-19281 & 9/11/05 & ND & ND & ND \\
\hline & & CNSB09-W-16318 & 10/11/05 & ND & ND & ND \\
\hline & & CNSB09-W-19902 & 3/17/06 & ND & ND & ND \\
\hline & & CNSB09-W-22502 & $9 / 25 / 06$ & ND & ND & ND \\
\hline & & CNSB09-W-16211 & 3/28/07 & ND & ND & ND \\
\hline & & CNSB09-W-16231 & $9 / 26 / 07$ & ND & ND & ND \\
\hline & & CNSB09-W-26033 & 3/20/08 & ND & ND & ND \\
\hline & & CNSB09-W-26688 & 9/10/08 & ND & ND & ND \\
\hline
\end{tabular}


TABLE 3.2 (Cont.)

\begin{tabular}{|c|c|c|c|c|c|c|}
\hline \multirow[b]{2}{*}{ Well } & \multirow[b]{2}{*}{$\begin{array}{l}\text { Screen } \\
\text { Interval } \\
\text { (ft BGL) }\end{array}$} & \multirow[b]{2}{*}{ Sample } & \multirow[b]{2}{*}{$\begin{array}{l}\text { Sampling } \\
\text { Date }\end{array}$} & \multicolumn{3}{|c|}{ Concentration $(\mu \mathrm{g} / \mathrm{L})$} \\
\hline & & & & $\begin{array}{c}\text { Carbon } \\
\text { Tetrachloride }\end{array}$ & Chloroform & $\begin{array}{c}\text { Methylene } \\
\text { Chloride }\end{array}$ \\
\hline \multicolumn{7}{|c|}{ Pilot test wells installed in September $2008^{e}$} \\
\hline PMP1 & $50-60$ & CNPMP1-W-26689 & 9/9/08 & 136 & 30 & ND \\
\hline PMP2 & $50-60$ & CNPMP2-W-26690 & 9/9/08 & 1854 & 318 & 5.6 \\
\hline PMP3 & $50-60$ & CNPMP3-W-26691 & 9/9/08 & 21 & 57 & 6.2 \\
\hline PMP4 & $48.75-58.75$ & CNPMP4-W-26692 & 9/9/08 & 49 & 4.2 & ND \\
\hline PMP5 & $50-60$ & CNPMP5-W-26693 & 9/10/08 & 418 & 46 & $1.6 \mathrm{~J}$ \\
\hline PMP6 & $50-60$ & CNPMP6-W-26694 & 9/8/08 & 110 & 7.8 & ND \\
\hline PMP7 & $50-60$ & CNPMP7-W-26695 & 9/9/08 & 119 & 13 & ND \\
\hline PMP8 & $50-60$ & CNPMP8-W-26696 & 9/9/08 & 72 & 125 & 3.4 \\
\hline PMP9 & $50-60$ & CNPMP9-W-26697 & 9/9/08 & 7.6 & $0.4 \mathrm{~J}$ & ND \\
\hline
\end{tabular}

a ND, not detected at an instrument detection limit of $0.1 \mu \mathrm{g} / \mathrm{L}$.

b Qualifier R indicates that the contaminant was present in the associated equipment rinsate.

c Qualifier J indicates an estimated concentration below the method quantitation limit of $1.0 \mu \mathrm{g} / \mathrm{L}$.

d Qualifier B indicates that the contaminant was present in the associated method blank.

e Details for the pilot test wells were reported separately (Argonne 2009). 
TABLE 3.3 Field measurements for groundwater samples collected at Centralia, August 2004 to September 2008.

\begin{tabular}{|c|c|c|c|c|c|c|c|c|c|}
\hline \multirow[b]{2}{*}{ Location } & \multirow{2}{*}{$\begin{array}{l}\text { Screen } \\
\text { Interval } \\
\text { (ft BGL) }\end{array}$} & \multirow[b]{2}{*}{$\begin{array}{l}\text { Sample } \\
\text { Date }\end{array}$} & \multirow{2}{*}{\multicolumn{2}{|c|}{ Temperature }} & \multirow[b]{2}{*}{$\begin{array}{l}\text { Conductivity } \\
\qquad(\mu \mathrm{S} / \mathrm{cm})\end{array}$} & \multicolumn{3}{|c|}{ Concentration (mg/L) } & \multirow[b]{2}{*}{$\begin{array}{l}\text { ORF } \\
(\mathrm{mV}\end{array}$} \\
\hline & & & & & & $\begin{array}{c}\text { Dissolved } \\
\text { Oxygen }\end{array}$ & $\begin{array}{l}\text { Carbon } \\
\text { Dioxide }\end{array}$ & Iron(II) & \\
\hline \multicolumn{10}{|c|}{ Wells in the approved sitewide monitoring network } \\
\hline \multirow[t]{9}{*}{ MW01 } & $54.5-64.5$ & $8 / 24 / 04$ & 16.3 & 7.39 & 652 & 0.06 & 25 & 0.00 & 230 \\
\hline & & 9/10/05 & 16.3 & 7.26 & 599 & 6.31 & $-\mathrm{a}$ & 0.00 & 104 \\
\hline & & 10/11/05 & 16.4 & 6.45 & 634 & - & - & - & - \\
\hline & & 3/15/06 & 14.3 & 7.56 & 621 & 9.33 & 30 & 0.04 & 297 \\
\hline & & 9/25/06 & 13.3 & 7.01 & 782 & 6.82 & 50 & 0.31 & 92 \\
\hline & & $3 / 29 / 07$ & 16.5 & 6.54 & 629 & 4.39 & - & 0.00 & 174 \\
\hline & & $9 / 26 / 07$ & 17.8 & 7.06 & 630 & 0.89 & 35 & 0.09 & 146 \\
\hline & & 3/19/08 & 9.5 & 7.31 & 613 & 3.34 & - & - & 122 \\
\hline & & 9/9/08 & 13.9 & 7.28 & 595 & 5.18 & 20 & 0.03 & 28 \\
\hline \multirow{9}{*}{ MW02 } & $49.5-59.5$ & $8 / 26 / 04$ & 14.4 & 7.31 & 729 & 0.16 & 20 & 0.12 & 235 \\
\hline & & $9 / 11 / 05$ & 15.3 & 7.02 & 739 & 1.28 & - & - & - \\
\hline & & $10 / 12 / 05$ & 14.8 & 6.60 & 766 & - & - & - & - \\
\hline & & 3/16/06 & 14.2 & 6.78 & 759 & 1.24 & - & 0.00 & 295 \\
\hline & & 9/26/06 & 13.2 & 6.98 & 957 & 3.05 & 40 & 0.06 & 67 \\
\hline & & $3 / 26 / 07$ & 15.7 & 6.39 & 739 & 2.29 & 50 & - & 67 \\
\hline & & 9/26/07 & 15.4 & 7.04 & 763 & 3.39 & 25 & 0.00 & 156 \\
\hline & & $3 / 12 / 08$ & 11.1 & 5.58 & 10174 & 0.28 & - & $3.3^{b}$ & -42 \\
\hline & & 9/8/08 & 13.1 & 6.12 & 6821 & 0.40 & 50 & $3.3^{b}$ & -74 \\
\hline \multirow[t]{9}{*}{ MW03 } & $50.5-60.5$ & $8 / 24 / 04$ & 13.1 & 7.28 & 783 & 0.10 & 55 & 0.21 & 230 \\
\hline & & 9/10/05 & 15.1 & 7.05 & 715 & 10.42 & 65 & 0.00 & 142 \\
\hline & & 10/11/05 & 16.3 & 6.46 & 765 & - & - & - & - \\
\hline & & 3/17/06 & 13.8 & 6.75 & 753 & 9.39 & 77 & 0.00 & 290 \\
\hline & & 9/26/06 & 13.2 & 6.92 & 960 & 11.57 & 45 & 0.08 & 251 \\
\hline & & $3 / 27 / 07$ & 15.3 & 6.40 & 774 & 7.73 & 25 & - & 268 \\
\hline & & $9 / 25 / 07$ & 14.3 & 6.97 & 738 & 8.44 & 30 & 0.00 & 162 \\
\hline & & $3 / 12 / 08$ & 14.6 & 7.12 & 777 & 7.90 & - & 3.13 & 89 \\
\hline & & $9 / 9 / 08$ & 14.9 & 7.13 & 763 & 9.60 & 110 & 0.12 & 66 \\
\hline \multirow[t]{9}{*}{ MW04 } & $37.5-47.5$ & $8 / 24 / 04$ & 16.2 & 7.39 & 717 & 0.11 & 40 & 0.04 & 210 \\
\hline & & 9/11/05 & 15.4 & 7.18 & 665 & 8.43 & 60 & 0.00 & 226 \\
\hline & & 10/11/05 & 14.4 & 7.14 & 811 & - & - & - & - \\
\hline & & 3/15/06 & 13.5 & 7.78 & 675 & 6.82 & 55 & 0.06 & 283 \\
\hline & & $9 / 25 / 06$ & - & 7.02 & 613 & 9.13 & 40 & 0.19 & 46 \\
\hline & & $3 / 28 / 07$ & 15.4 & 6.47 & 678 & 5.46 & - & 0.00 & 197 \\
\hline & & $9 / 24 / 07$ & 17.4 & 7.10 & 667 & 6.94 & 35 & 0.24 & 261 \\
\hline & & 3/19/08 & 11.2 & 7.32 & 636 & 7.55 & - & - & 164 \\
\hline & & $9 / 9 / 08$ & 14.2 & 7.14 & 648 & 8.68 & 100 & 0.00 & 72 \\
\hline \multirow[t]{9}{*}{ MW05 } & $34.5-44.5$ & $8 / 25 / 04$ & 14.3 & 7.14 & 613 & 0.08 & 25 & 0.06 & 215 \\
\hline & & 9/10/05 & 14.2 & 6.80 & 620 & 1.40 & 110 & 0.00 & 160 \\
\hline & & 10/11/05 & 14.8 & 6.35 & 610 & - & - & - & - \\
\hline & & 3/15/06 & 14.3 & 6.90 & 701 & 0.90 & 30 & 0.06 & 156 \\
\hline & & $9 / 25 / 06$ & 13.6 & 6.95 & 768 & 0.09 & 50 & 0.02 & 55 \\
\hline & & $3 / 28 / 07$ & 14.4 & 6.44 & 573 & 4.53 & 35 & 0.00 & 295 \\
\hline & & $9 / 24 / 07$ & 15.8 & 7.06 & 368 & 3.09 & 45 & 0.00 & 182 \\
\hline & & 3/19/08 & 12.9 & 7.42 & 642 & 5.42 & - & - & 177 \\
\hline & & 9/10/08 & 13.9 & 7.11 & 663 & 7.14 & 95 & 0.00 & 130 \\
\hline
\end{tabular}


TABLE 3.3 (Cont.)

\begin{tabular}{|c|c|c|c|c|c|c|c|c|c|}
\hline \multirow[b]{2}{*}{ Location } & \multirow{2}{*}{$\begin{array}{l}\text { Screen } \\
\text { Interval } \\
\text { (ft BGL) }\end{array}$} & \multirow[b]{2}{*}{$\begin{array}{l}\text { Sample } \\
\text { Date }\end{array}$} & \multirow{2}{*}{\multicolumn{2}{|c|}{ Temperature }} & \multirow[b]{2}{*}{$\begin{array}{l}\text { Conductivity } \\
(\mu S / \mathrm{cm})\end{array}$} & \multicolumn{3}{|c|}{ Concentration (mg/L) } & \multirow[b]{2}{*}{$\begin{array}{l}\text { ORF } \\
(\mathrm{mV}\end{array}$} \\
\hline & & & & & & $\begin{array}{l}\text { Dissolved } \\
\text { Oxygen }\end{array}$ & $\begin{array}{l}\text { Carbon } \\
\text { Dioxide }\end{array}$ & Iron(II) & \\
\hline \multicolumn{10}{|c|}{ Wells in the approved sitewide monitoring network (cont.) } \\
\hline \multirow[t]{9}{*}{ MW06 } & $46.5-56.5$ & $8 / 25 / 04$ & 15.9 & 7.50 & 637 & 0.05 & 15 & 0.00 & 215 \\
\hline & & 9/10/05 & 14.6 & 7.23 & 659 & 0.04 & 60 & 0.00 & 41 \\
\hline & & $10 / 11 / 05$ & 15.8 & 6.99 & 638 & - & - & - & - \\
\hline & & $3 / 15 / 06$ & 14.1 & 7.38 & 630 & 9.87 & 35 & 0.02 & 263 \\
\hline & & $9 / 27 / 06$ & 13.1 & 6.16 & 652 & 0.05 & 45 & 1.12 & 63 \\
\hline & & $3 / 27 / 07$ & 19.0 & 6.42 & 466 & 0.11 & 20 & 0.00 & 13 \\
\hline & & 9/24/07 & 16.8 & 7.11 & 463 & 8.00 & 25 & 0.41 & 191 \\
\hline & & 3/19/08 & 14.1 & 7.01 & 552 & 7.00 & - & - & 172 \\
\hline & & 9/9/08 & 14.4 & 7.20 & 437 & 0.36 & 105 & 0.07 & -96 \\
\hline \multirow[t]{6}{*}{ MW07 } & $45-55$ & $3 / 14 / 06$ & 14.7 & 6.61 & 709 & 0.34 & - & 0.03 & 143 \\
\hline & & 9/26/06 & 13.1 & 7.23 & 642 & 2.91 & 50 & 0.00 & - \\
\hline & & $3 / 26 / 07$ & 15.8 & 6.50 & 642 & 1.87 & 30 & 0.00 & 261 \\
\hline & & $9 / 24 / 07$ & 19.0 & 7.18 & 609 & 9.05 & 60 & 0.18 & 190 \\
\hline & & 3/19/08 & 12.5 & 7.29 & 647 & 2.70 & - & - & 215 \\
\hline & & 9/9/08 & 15.6 & 7.10 & 629 & 1.41 & 68 & 0.00 & 16 \\
\hline \multirow[t]{6}{*}{ MW08 } & $38-53$ & $3 / 14 / 06$ & 13.5 & 6.35 & 854 & 5.32 & - & 0.00 & 145 \\
\hline & & 9/26/06 & 13.3 & 6.75 & 1095 & 0.16 & 50 & 0.18 & 37 \\
\hline & & $3 / 27 / 07$ & 15.8 & 6.31 & 874 & 1.49 & 30 & 0.21 & 237 \\
\hline & & $9 / 25 / 07$ & 15.8 & 6.92 & 627 & 1.42 & 45 & 0.14 & 219 \\
\hline & & $3 / 20 / 08$ & 13.5 & 7.19 & 869 & 2.11 & - & - & 185 \\
\hline & & 9/10/08 & 16.3 & 7.03 & 864 & 1.17 & 100 & 0.03 & 117 \\
\hline \multirow[t]{6}{*}{ MW09 } & $25-35$ & $3 / 15 / 06$ & 17.7 & 7.33 & 664 & 0.95 & 55 & 0.09 & 214 \\
\hline & & $9 / 25 / 06$ & 12.8 & 6.87 & 859 & 1.59 & 45 & 0.18 & 90 \\
\hline & & $3 / 27 / 07$ & 14.9 & 6.35 & 689 & 4.10 & 30 & 0.69 & 152 \\
\hline & & $9 / 24 / 07$ & 16.6 & 6.94 & 1999 & 3.86 & 55 & 0.14 & 186 \\
\hline & & $3 / 20 / 08$ & 13.5 & 7.17 & 720 & 4.70 & - & - & 173 \\
\hline & & 9/10/08 & 14.7 & 7.02 & 706 & 3.68 & 110 & 0.07 & 120 \\
\hline \multirow[t]{6}{*}{ MW10 } & $30-45$ & $3 / 14 / 06$ & 14.8 & 6.60 & 834 & 6.42 & 65 & 0.00 & 166 \\
\hline & & 9/26/06 & 13.6 & 6.87 & 1058 & 6.94 & 50 & 0.50 & 51 \\
\hline & & $3 / 28 / 07$ & 17.0 & 6.36 & 834 & 5.09 & 35 & 0.00 & 270 \\
\hline & & $9 / 25 / 07$ & 15.8 & 6.94 & 827 & 6.64 & 35 & 0.21 & 199 \\
\hline & & $3 / 20 / 08$ & 10.9 & 7.18 & 898 & 6.12 & - & - & 187 \\
\hline & & 9/9/08 & 14.8 & 7.05 & 879 & 7.18 & 100 & 0.06 & 94 \\
\hline \multirow[t]{9}{*}{ SB01 } & $40-50$ & 8/26/04 & 26.0 & 7.46 & 699 & 5.21 & 30 & 0.00 & 210 \\
\hline & & 9/9/05 & 25.0 & 7.11 & 674 & 6.25 & 95 & 0.00 & 140 \\
\hline & & $10 / 12 / 05$ & 13.8 & 7.23 & 686 & - & - & - & - \\
\hline & & $3 / 17 / 06$ & 12.4 & 7.30 & 692 & 5.98 & 55 & 0.00 & 185 \\
\hline & & 9/27/06 & 14.4 & 7.03 & 832 & 6.54 & 40 & 0.52 & 198 \\
\hline & & $3 / 27 / 07$ & 18.0 & 6.37 & 659 & 3.81 & 25 & 0.23 & 173 \\
\hline & & $9 / 27 / 07$ & 13.5 & 7.24 & 720 & 6.55 & 45 & 1.04 & 143 \\
\hline & & 3/20/08 & 15.6 & 7.29 & 783 & 8.02 & - & - & 182 \\
\hline & & 9/10/08 & 16.5 & 7.10 & 676 & 2.89 & 100 & 0.17 & 100 \\
\hline
\end{tabular}


TABLE 3.3 (Cont.)

\begin{tabular}{|c|c|c|c|c|c|c|c|c|c|}
\hline \multirow[b]{2}{*}{ Location } & \multirow{2}{*}{$\begin{array}{l}\text { Screen } \\
\text { Interval } \\
\text { (ft BGL) }\end{array}$} & \multirow[b]{2}{*}{$\begin{array}{l}\text { Sample } \\
\text { Date }\end{array}$} & \multirow{2}{*}{\multicolumn{2}{|c|}{ Temperature }} & \multirow[b]{2}{*}{$\begin{array}{l}\text { Conductivity } \\
\qquad(\mu \mathrm{S} / \mathrm{cm})\end{array}$} & \multicolumn{3}{|c|}{ Concentration (mg/L) } & \multirow[b]{2}{*}{$\begin{array}{l}\text { ORF } \\
(\mathrm{mV}\end{array}$} \\
\hline & & & & & & $\begin{array}{l}\text { Dissolved } \\
\text { Oxygen }\end{array}$ & $\begin{array}{l}\text { Carbon } \\
\text { Dioxide }\end{array}$ & Iron(II) & \\
\hline \multicolumn{10}{|c|}{ Wells in the approved sitewide monitoring network (cont.) } \\
\hline \multirow[t]{9}{*}{ SB04 } & $51-61$ & $8 / 26 / 04$ & 17.9 & 7.14 & 765 & 3.78 & 55 & 0.37 & 230 \\
\hline & & 9/9/05 & 16.0 & 7.09 & 708 & 8.67 & 100 & - & 206 \\
\hline & & $10 / 12 / 05$ & 13.9 & 7.17 & 813 & - & - & - & - \\
\hline & & $3 / 16 / 06$ & 13.0 & 7.57 & 799 & 5.96 & 30 & - & 276 \\
\hline & & $9 / 25 / 06$ & 14.9 & 7.16 & 791 & 9.32 & 70 & 1.18 & 64 \\
\hline & & $3 / 28 / 07$ & 16.2 & 6.45 & 850 & 6.18 & - & 0.23 & 266 \\
\hline & & $9 / 26 / 07$ & 19.8 & 7.03 & 760 & 6.61 & 30 & 0.00 & 202 \\
\hline & & $3 / 12 / 08$ & 15.5 & 7.04 & 819 & 6.16 & - & 0.09 & 154 \\
\hline & & 9/9/08 & 16.5 & 7.11 & 802 & 6.48 & 100 & 0.02 & 70 \\
\hline \multirow[t]{9}{*}{ SB05 } & $32-42$ & $8 / 26 / 04$ & 15.7 & 7.25 & 761 & - & 25 & 0.06 & 220 \\
\hline & & 9/9/05 & 16.9 & 6.98 & 687 & 7.58 & 100 & - & - \\
\hline & & $10 / 12 / 05$ & 14.0 & 7.00 & 728 & - & - & - & - \\
\hline & & $3 / 17 / 06$ & 13.3 & 7.67 & 718 & 4.80 & 40 & 0.18 & 253 \\
\hline & & $9 / 27 / 06$ & 13.7 & 6.58 & 763 & 4.70 & 50 & 0.25 & 78 \\
\hline & & $3 / 28 / 07$ & 16.7 & 4.03 & 0.11 & 2.58 & 35 & 0.07 & 296 \\
\hline & & $9 / 26 / 07$ & 15.1 & 6.98 & 810 & 4.10 & 30 & 0.50 & 221 \\
\hline & & 3/20/08 & 14.5 & 7.11 & 870 & 5.56 & - & - & 206 \\
\hline & & 9/9/08 & 13.7 & 6.79 & 890 & 7.60 & 90 & 0.09 & 56 \\
\hline \multirow[t]{6}{*}{ SB07R } & $45-60$ & $3 / 15 / 06$ & 16.8 & 7.24 & 685 & 7.41 & 60 & 0.08 & 83 \\
\hline & & $9 / 26 / 06$ & 13.2 & 6.89 & 842 & 6.17 & 55 & 0.26 & 67 \\
\hline & & $3 / 26 / 07$ & 19.0 & 6.38 & 668 & 5.08 & 40 & 0.07 & 237 \\
\hline & & $9 / 25 / 07$ & 17.4 & 7.06 & 642 & 6.30 & 35 & 0.11 & 170 \\
\hline & & 3/12/08 & 17.3 & 7.18 & 639 & 5.33 & - & 0.00 & 108 \\
\hline & & 9/9/08 & 14.1 & 7.06 & 631 & 5.08 & 100 & 0.07 & 55 \\
\hline \multirow[t]{9}{*}{ SB08 } & $52-62$ & 8/26/04 & 19.5 & 7.31 & 635 & 0.16 & 20 & 0.53 & 235 \\
\hline & & 9/8/05 & 21.2 & 7.27 & 598 & 3.21 & 75 & 0.00 & 111 \\
\hline & & $10 / 12 / 05$ & 13.9 & 7.15 & 630 & - & - & - & - \\
\hline & & $3 / 17 / 06$ & 12.9 & 7.14 & 645 & 3.40 & 40 & 0.00 & 246 \\
\hline & & $9 / 21 / 06$ & 14.1 & 6.96 & 809 & 4.53 & 40 & 0.00 & 37 \\
\hline & & $3 / 28 / 07$ & 15.8 & 6.53 & 645 & 3.57 & 35 & 0.24 & 208 \\
\hline & & $9 / 26 / 07$ & 17.4 & 7.11 & 617 & 4.56 & 40 & 0.77 & 156 \\
\hline & & $3 / 12 / 08$ & 17.1 & 7.17 & 642 & 3.63 & - & 0.14 & 102 \\
\hline & & 9/8/08 & 13.6 & 7.14 & 626 & 2.70 & 90 & 0.00 & 230 \\
\hline \multirow[t]{9}{*}{ SB09 } & $32-42$ & 8/26/04 & 30.9 & 7.09 & 910 & 0.26 & 75 & 0.00 & 185 \\
\hline & & 9/11/05 & 14.6 & 6.71 & 877 & 0.13 & 225 & 0.00 & - \\
\hline & & 10/11/05 & 13.9 & 6.85 & 910 & - & - & - & - \\
\hline & & $3 / 17 / 06$ & 11.7 & 7.03 & 969 & 1.53 & 99 & 0.00 & 206 \\
\hline & & $9 / 25 / 06$ & 14.2 & 7.00 & 976 & 0.29 & 70 & 0.38 & 86 \\
\hline & & $3 / 28 / 07$ & 14.3 & 6.32 & 957 & 0.89 & 40 & 0.09 & 236 \\
\hline & & 9/26/07 & 15.2 & 6.77 & 969 & 1.53 & 45 & 0.12 & 199 \\
\hline & & 3/20/08 & 10.1 & 6.94 & 1000 & 1.57 & - & - & 221 \\
\hline & & 9/10/08 & 18.4 & 6.87 & 977 & 0.56 & 160 & 0.11 & 109 \\
\hline
\end{tabular}


TABLE 3.3 (Cont.)

\begin{tabular}{|c|c|c|c|c|c|c|c|c|c|}
\hline \multirow[b]{2}{*}{ Location } & \multirow{2}{*}{$\begin{array}{l}\text { Screen } \\
\text { Interval } \\
\text { (ft BGL) }\end{array}$} & \multirow[b]{2}{*}{$\begin{array}{l}\text { Sample } \\
\text { Date }\end{array}$} & \multirow{2}{*}{\multicolumn{2}{|c|}{ Temperature }} & \multirow[b]{2}{*}{$\begin{array}{l}\text { Conductivity } \\
(\mu S / \mathrm{cm})\end{array}$} & \multicolumn{3}{|c|}{ Concentration (mg/L) } & \multirow[b]{2}{*}{$\begin{array}{l}\text { ORP } \\
(\mathrm{mV})\end{array}$} \\
\hline & & & & & & $\begin{array}{l}\text { Dissolved } \\
\text { Oxygen }\end{array}$ & $\begin{array}{l}\text { Carbon } \\
\text { Dioxide }\end{array}$ & Iron(II) & \\
\hline \multicolumn{10}{|c|}{ Pilot test wells installed in September $2008^{c}$} \\
\hline PMP1 & $50-60$ & 9/9/08 & 14.4 & 5.54 & 700 & 1.37 & 115 & 0.23 & 40 \\
\hline PMP2 & $50-60$ & 9/9/08 & 14.4 & 7.09 & 997 & 0.05 & 180 & 1.68 & -41 \\
\hline PMP3 & $50-60$ & 9/9/08 & 14.5 & 6.98 & 1301 & 0.03 & 150 & $3.3^{b}$ & -150 \\
\hline PMP4 & $48.75-58.75$ & 9/9/08 & 14.3 & 4.97 & 738 & 4.87 & 100 & 0.49 & 134 \\
\hline PMP5 & $50-60$ & 9/10/08 & 16.9 & 7.20 & 875 & 2.51 & 105 & 0.18 & 117 \\
\hline PMP6 & $50-60$ & 9/8/08 & 13.2 & 6.87 & 787 & 3.32 & 75 & 0.09 & 173 \\
\hline PMP7 & $50-60$ & 9/9/08 & 14.2 & 6.30 & 807 & 2.18 & 70 & 0.18 & 15 \\
\hline PMP8 & $50-60$ & 9/9/08 & 14.4 & 7.05 & 1388 & 0.03 & 60 & 2.72 & -129 \\
\hline PMP9 & $50-60$ & 9/9/08 & 14.0 & 6.36 & 606 & 7.78 & 120 & 0.10 & 45 \\
\hline
\end{tabular}

\footnotetext{
a Measurement not recorded.

b Maximum reading from instrument.

c Details for the pilot test wells were reported separately (Argonne 2009).
} 


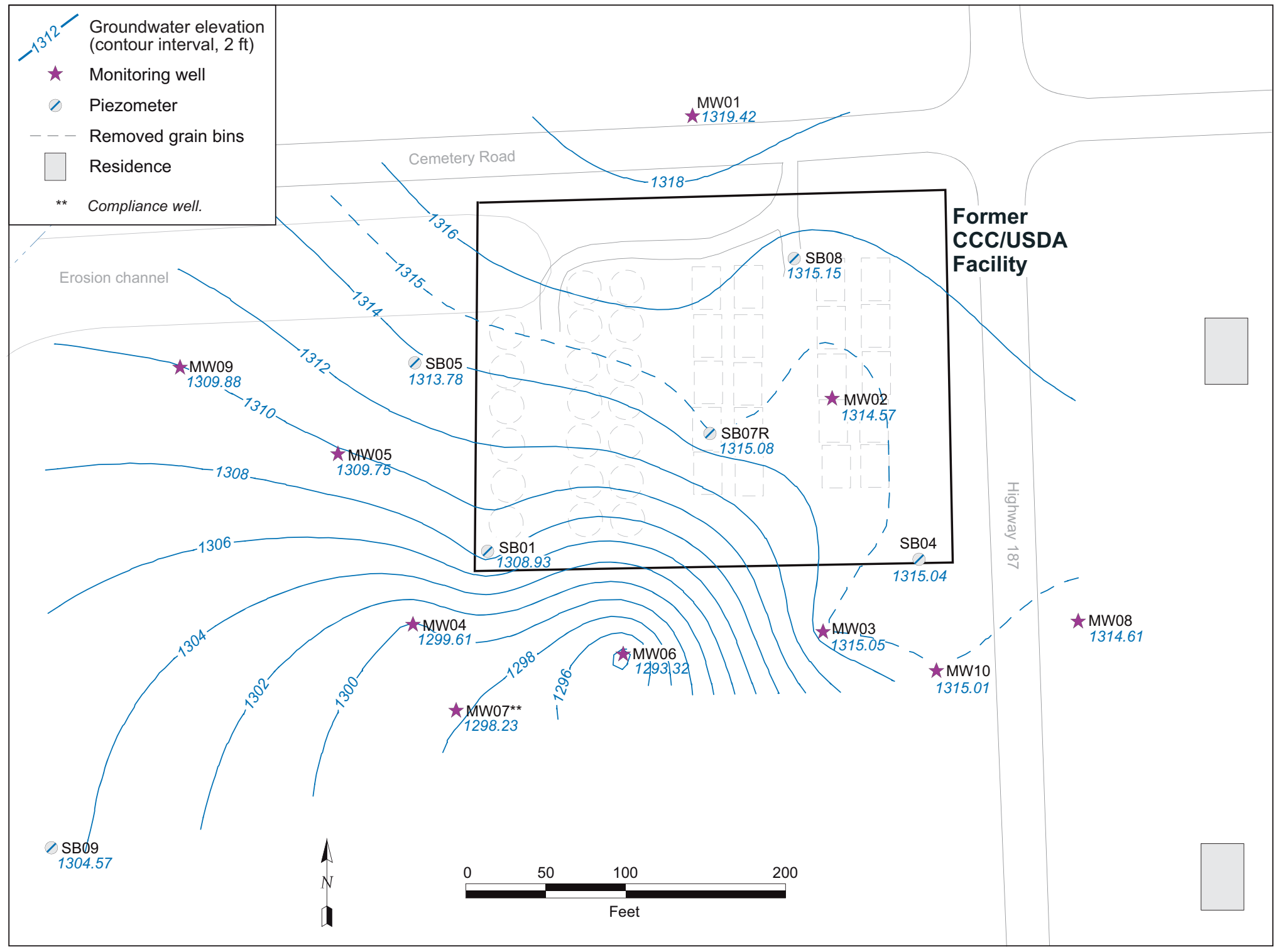

FIGURE 3.1 Potentiometric surface at Centralia, based on water levels measured manually on June 25, 2008. 


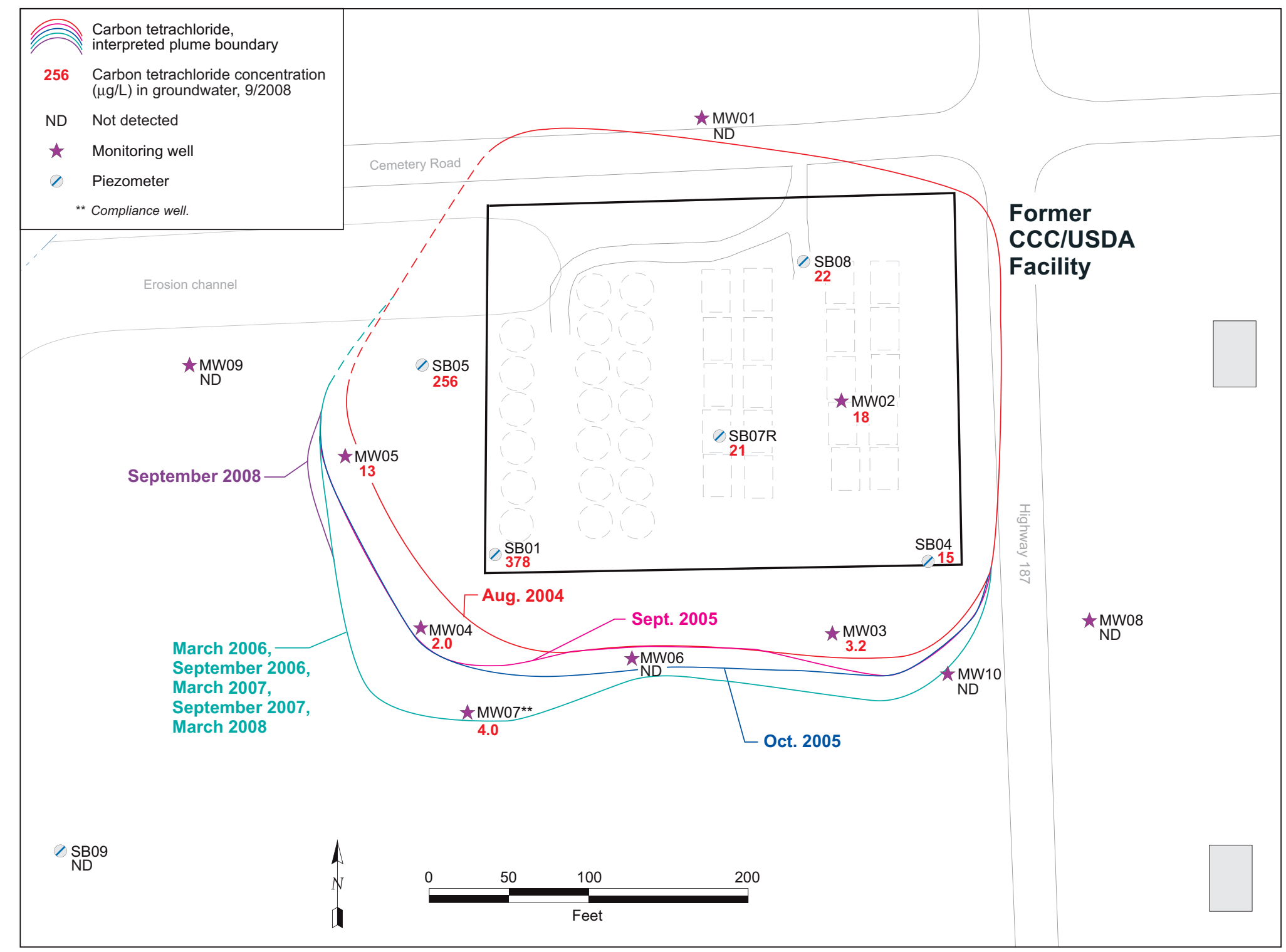

FIGURE 3.2 Carbon tetrachloride levels in groundwater at Centralia in September 2008, with the interpreted lateral extent of the contaminant at intervals during the period August 2004 to September 2008. 


\section{Conclusions and Recommendations}

\subsection{Conclusions}

The findings of the September 2008 monitoring event at Centralia support the following conclusions:

- Measurements of groundwater levels obtained manually and through the use of automatic recorders have consistently indicated an apparent direction of groundwater flow to the south-southwest across the former CCC/USDA facility.

- The September 2008 carbon tetrachloride data for monitoring wells in the approved sitewide network are generally consistent with previous results. Longer-term trends of slightly increasing carbon tetrachloride concentrations continue to be observed at monitoring points SB05, MW03, MW04, and MW07, along the western and southern margins of the groundwater plume. These trends suggest very slow expansion of the plume at the downgradient locations.

- A dramatic decrease in carbon tetrachloride concentration at MW02, from $1,138 \mu \mathrm{g} / \mathrm{L}$ in September 2007, followed implementation of the interim measure pilot test in December 2007. A reduction of 96-99\% in the levels of carbon tetrachloride in groundwater in the injection area near MW02 was observed within the first 5-7 weeks after injection. Monitoring at MW02 in March 2008 showed no carbon tetrachloride present, while monitoring in September 2008 indicated that carbon tetrachloride concentrations had increased slightly (to $18 \mu \mathrm{g} / \mathrm{L}$ ), though they remained at or near the dramatically decreased levels observed initially post-injection.

- Sampling in 2004-2007 yielded only limited evidence for the presence of subsurface conditions at Centralia conducive to anaerobic degradation of the carbon tetrachloride contamination in groundwater. Nevertheless, consistent detection of chloroform at relatively elevated concentrations at monitoring 
points SB01, SB05, and MW02 suggested that degradation of carbon tetrachloride was occurring at those locations.

\subsection{Recommendations}

The September 2007 sampling was the final monitoring event performed under the original two-year, twice yearly monitoring program (Argonne 2005b) approved by the KDHE (2005a,b). The CCC/USDA developed an Interim Measure Conceptual Design (Argonne 2007b), proposing a pilot test of the Adventus EHC in situ chemical reduction (ISCR) technology, that was approved by the KDHE in November 2007 (KDHE 2007). Implementation of the interim measure occurred in December 2007. The results of the September 2007 monitoring (Argonne 2008a) served as pre-injection baseline data for existing monitoring points, per agreement with the KDHE.

The EHC materials, proprietary mixtures of food-grade organic carbon and zero-valent iron, were injected into the subsurface as a slurry (EHC) or in dissolved form (EHC-A) for subsequent slow release into the formation. The materials are designed to create highly reducing geochemical conditions in the vadose and saturated zones that foster both thermodynamic and biological reductive dechlorination of carbon tetrachloride. Adventus has estimated that the EHC materials might have an effect on the subsurface geochemical environment in the pilot test area for 1-5 years (Adventus 2009). The results to date of the EHC pilot injections were discussed with the KDHE in September 2008 and were documented separately (Argonne 2009). On the basis of these data, the CCC/USDA has recommended additional monitoring of the pilot test site to determine the longer-term effects of the EHC injections; however, no further implementation of the ISCR technology is proposed (Argonne 2009).

The March and September 2008 sampling events were extensions of the original two-year (from 2005 to 2007) program of twice yearly monitoring at Centralia previously approved by the KDHE. The KDHE (2008a) has requested that sitewide monitoring continue at Centralia until a final remedy has been selected (as part of a Corrective Action Study [CAS] evaluation) and implemented. The CCC/USDA has therefore recommended (Argonne 2009) the development of a revised monitoring program, to address both the continued pilot test and sitewide monitoring objectives. With the KDHE's approval, the interim plan presented here will be implemented while a CAS for the Centralia site is being prepared. 
The interim monitoring plan being recommended by the CCC/USDA acknowledges the observation that the contaminant plume has shown almost no movement or expansion during several years of monitoring. The elements of the proposed interim monitoring plan are as follows:

- Annual sampling of

- Twelve previously established (before the pilot test) monitoring points (locations identified in Figure 4.1) and

- The five outlying pilot test monitoring points (PMP4, PMP5, PMP6, PMP7, PMP9; Figure 4.2).

- Sampling twice yearly at the five pilot test monitoring points inside the injection area (PMP1, PMP2, PMP3, PMP8, MW02; Figure 4.2).

- Elimination of MW01 (upgradient), MW08 (upgradient), and SB09 (far downgradient) from the monitoring network, unless site conditions change to indicate the need for resumed monitoring at those locations. 


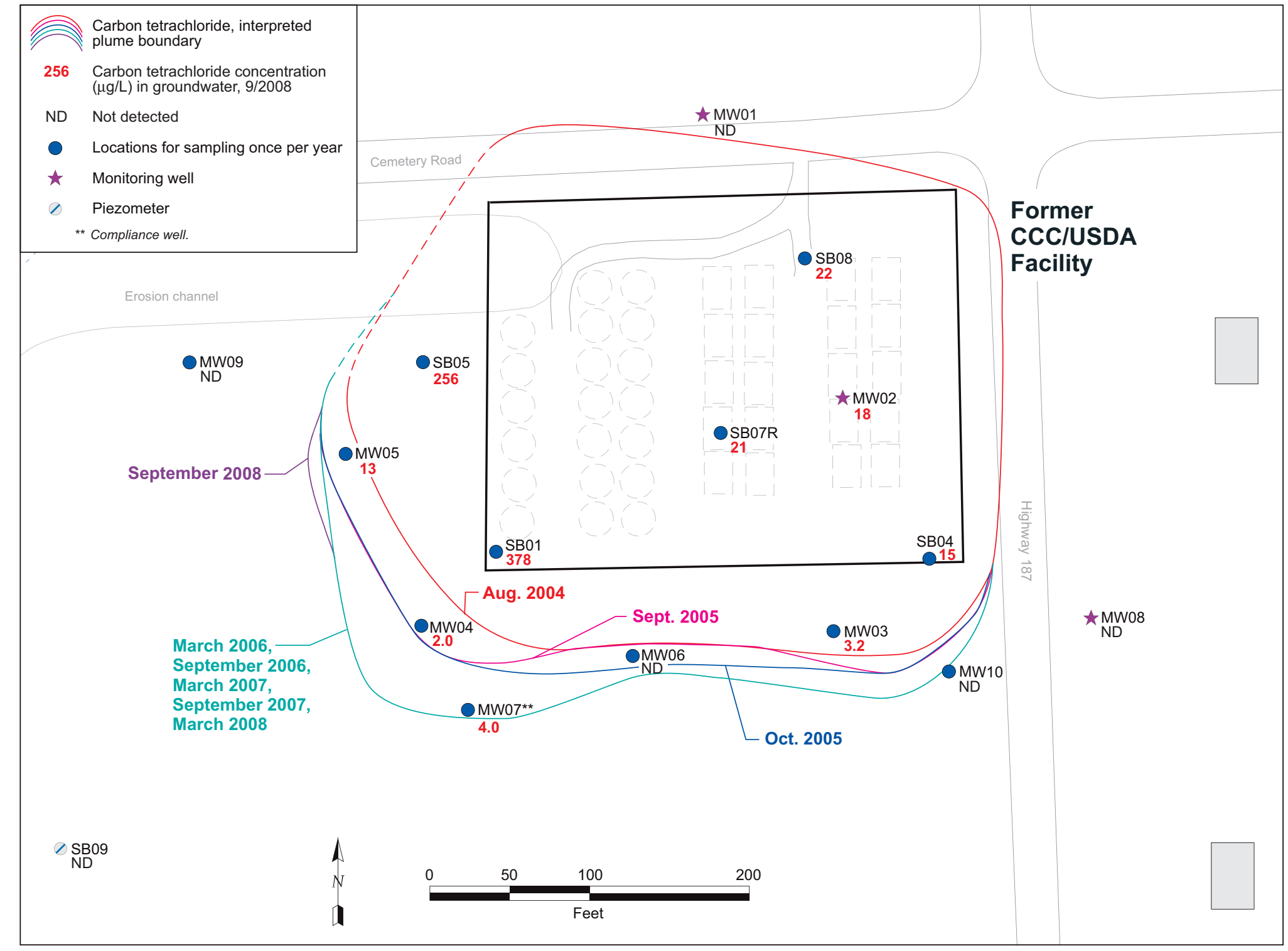

FIGURE 4.1 Previously established (before the pilot test) monitoring points proposed for ongoing annual monitoring. 


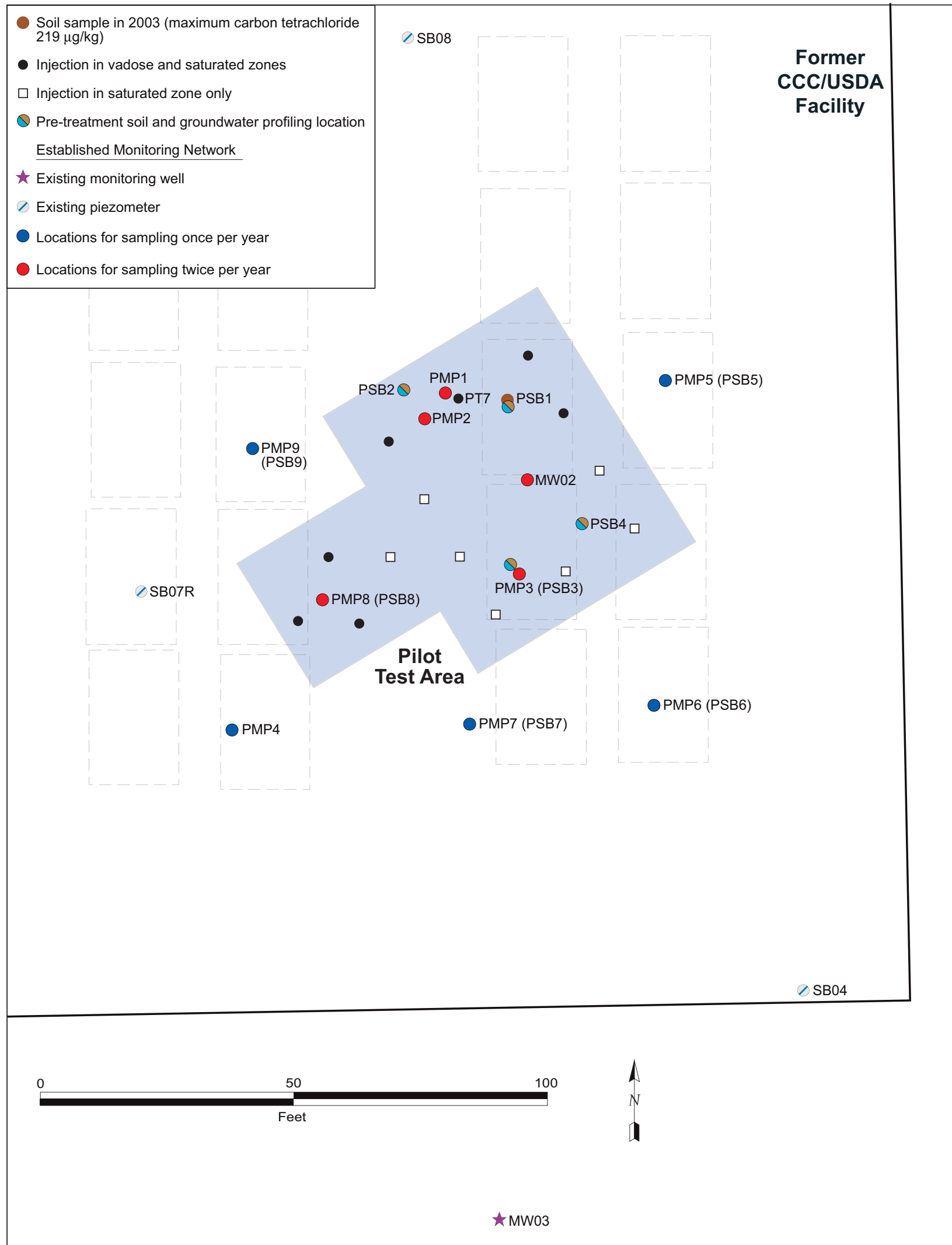

FIGURE 4.2 Pilot test monitoring points proposed for ongoing annual monitoring or twice yearly monitoring. 


\section{References}

Adventus, 2009, EHC ${ }^{\circledR}$ Technology Overview - Field Applications, Adventus Americas, Inc., Freeport, Illinois, http://www.adventusgroup.com/pdfs/EHC_applications.pdf, accessed January 15.

Argonne, 2002, Final Master Work Plan: Environmental Investigations at Former CCC/USDA Facilities in Kansas, 2002 Revision, ANL/ER/TR-02/2004, prepared for the Commodity Credit Corporation, U.S. Department of Agriculture, Washington, D.C., by Argonne National Laboratory, Argonne, Illinois, December.

Argonne, 2003, Final Phase I Report and Phase II Work Plan: QuickSite ${ }^{\circledR}$ Investigation, Centralia, Kansas, ANL/ER/TR-02/009, prepared for the Commodity Credit Corporation, U.S. Department of Agriculture, Washington, D.C., by Argonne National Laboratory, Argonne, Illinois, March.

Argonne, 2004, Final Phase II Report: QuickSite ${ }^{\circledR}$ Investigation, Centralia, Kansas, ANL/ER/TR-03/006, prepared for the Commodity Credit Corporation, U.S. Department of Agriculture, Washington, D.C., by Argonne National Laboratory, Argonne, Illinois, March.

Argonne, 2005a, Final Report: 2004 Monitoring Well Installation and Sampling at Centralia, Kansas, ANL/ER/TR-04/011, prepared for the Commodity Credit Corporation, U.S. Department of Agriculture, Washington, D.C., by Argonne National Laboratory, Argonne, Illinois, October.

Argonne, 2005b, Final Work Plan: Groundwater Monitoring at Centralia, Kansas, ANL/ER/TR-05/004, prepared for the Commodity Credit Corporation, U.S. Department of Agriculture, Washington, D.C., by Argonne National Laboratory, Argonne, Illinois, August.

Argonne, 2006a, Final Report: Groundwater Monitoring at Centralia, Kansas, in SeptemberOctober 2005 and March 2006, with Expansion of the Monitoring Network in January 2006, ANL/EVS/AGEM/TR-06-06, prepared for the Commodity Credit Corporation, U.S. Department of Agriculture, Washington, D.C., by Argonne National Laboratory, Argonne, Illinois, October.

Argonne, 2006b, September 2006 Monitoring Results for Centralia, Kansas, ANL/EVS/AGEM/CHRON-1003, prepared for the Commodity Credit Corporation, 
U.S. Department of Agriculture, Washington, D.C., by Argonne National Laboratory, Argonne, Illinois, November 10.

Argonne, 2007a, March 2007 Monitoring Results for Centralia, Kansas, ANL/EVS/AGEM/TR07-08, prepared for the Commodity Credit Corporation, U.S. Department of Agriculture, Washington, D.C., by Argonne National Laboratory, Argonne, Illinois, June.

Argonne, 2007b, Interim Measure Conceptual Design for Remediation at the Former CCC/USDA Grain Storage Facility at Centralia, Kansas: Pilot Test and Remedy Implementation, ANL/EVS/AGEM/TR-07-11, prepared for the Commodity Credit Corporation, U.S. Department of Agriculture, Washington, D.C., by Argonne National Laboratory, Argonne, Illinois, October.

Argonne, 2008a, September 2007 Monitoring Results for Centralia, Kansas, ANL/EVS/AGEM/TR-08-01, prepared for the Commodity Credit Corporation, U.S. Department of Agriculture, Washington, D.C., by Argonne National Laboratory, Argonne, Illinois, January.

Argonne, 2008b, March 2008 Monitoring Results for Centralia, Kansas, ANL/EVS/AGEM/TR08-08, prepared for the Commodity Credit Corporation, U.S. Department of Agriculture, Washington, D.C., by Argonne National Laboratory, Argonne, Illinois, May.

Argonne, 2009, Progress Report and Technical Evaluation of the ISCR Pilot Test Conducted at the Former CCC/USDA Grain Storage Facility in Centralia, Kansas, ANL/EVS/AGEM/TR-0818, prepared for the Commodity Credit Corporation, U.S. Department of Agriculture, Washington, D.C., by Argonne National Laboratory, Argonne, Illinois, January.

EPA, 1995, Method 524.2: Measurement of Purgeable Organic Compounds in Water by Capillary Column Gas Chromatography/Mass Spectrometry, Revision 4.1, edited by J.W. Munch, National Exposure Research Laboratory, Office of Research and Development, U.S. Environmental Protection Agency, Cincinnati, Ohio.

EPA, 1998, Technical Protocol for Evaluating Natural Attenuation of Chlorinated Solvents in Ground Water, EPA/600/R-98/128, Office of Research and Development, U.S. Environmental Protection Agency, Washington, D.C., September (http://www.epa.gov/superfund/resources/ gwdocs/protocol.htm). 
KDHE, 2001, Monitored Natural Attenuation, Policy BER-RS-042, Bureau of Environmental Remediation, Kansas Department of Health and Environment, Topeka, Kansas, March 30 (revised December 18, 2005; http://www.kdheks.gov/ber/policies/BER_RS_042.pdf).

KDHE, 2005a, letter from C. Carey (Bureau of Environmental Remediation, Kansas Department of Health and Environment, Topeka, Kansas) to C. Roe (Commodity Credit Corporation, U.S. Department of Agriculture, Washington, D.C.), regarding Final Work Plan: Groundwater Monitoring at Centralia, Kansas, October 13.

KDHE, 2005b, letter from C. Carey (Bureau of Environmental Remediation, Kansas Department of Health and Environment, Topeka, Kansas) to C. Roe (Commodity Credit Corporation, U.S. Department of Agriculture, Washington, D.C.), regarding results of the fall 2005 sampling at Centralia and recommendations for expansion of the monitoring network, November 22.

KDHE, 2007, letter from C. Carey (Bureau of Environmental Remediation, Kansas Department of Health and Environment, Topeka, Kansas) to C. Roe (Commodity Credit Corporation, U.S. Department of Agriculture, Washington, D.C.), regarding review of Interim Measure Conceptual Design for Centralia, Kansas, November 9.

KDHE, 2008a, letter from C. Carey (Bureau of Environmental Remediation, Kansas Department of Health and Environment, Topeka, Kansas) to C. Roe (Commodity Credit Corporation, U.S. Department of Agriculture, Washington, D.C.), regarding September 2007 Monitoring Results for Centralia, Kansas, March 12.

KDHE, 2008b, electronic mail message from C. Carey (Bureau of Environmental Remediation, Kansas Department of Health and Environment, Topeka, Kansas) to L. LaFreniere (Argonne National Laboratory, Argonne, Illinois), regarding use of the low-flow sampling technique at Centralia, February 11.

Puls, R.W., and Barcelona, M.J., 1996, "Low-Flow (Minimal Drawdown) Ground-Water Sampling Procedures,” EPA/540/S-95/504, in Ground Water Issue, Superfund Technology Support Center for Ground Water, National Risk Management Research Laboratory, Ada, Oklahoma, April (www.epa.gov/tio/tsp/download/lwflw2a.pdf). 


\section{Appendix A:}

Sequence of Sampling Activities at Centralia, Kansas, in September 2008 
TABLE A.1 Sequence of water sampling activities at Centralia in September 2008.

\begin{tabular}{|c|c|c|c|c|c|c|c|}
\hline Date & Time & Sample & Type $^{a}$ & Location $^{b}$ & $\begin{array}{l}\text { Depthc } \\
\text { (ft TOC) }\end{array}$ & $\begin{array}{l}\text { Chain of } \\
\text { Custody }\end{array}$ & Sample Description \\
\hline 9/8/08 & $12: 04$ & CNSB08-W-26687 & $\mathrm{CPT} / \mathrm{P}$ & SB08 & $52-62$ & 6122 & $\begin{array}{l}\text { Depth to water }=18.6 \mathrm{ft} \text {. Depth of } 1 \text {-in. well }=62 \mathrm{ft} \text {. Sample } \\
\text { collected by using low-flow bladder pump after purging of } 3 \mathrm{~L} \text {. } \\
\text { Water clear. }\end{array}$ \\
\hline 9/8/08 & 13:16 & CNMW02-W-26674 & MW & MW02 & $49.5-59.5$ & 6122 & $\begin{array}{l}\text { Depth to water }=21.9 \mathrm{ft} \text {. Depth of } 4 \text {-in. well }=59.5 \mathrm{ft} \text {. Sample } \\
\text { collected by using low-flow bladder pump after purging of } \\
6.7 \mathrm{~L} \text {. Water tannish in color with strong manure-like odor. } \\
\text { Carbon dioxide difficult to read. }\end{array}$ \\
\hline 9/8/08 & $13: 19$ & CNPMP6-W-26694 & MW & PMP6 & $50-60$ & 6124 & $\begin{array}{l}\text { Depth to water }=20.9 \mathrm{ft} \text {. Depth of } 0.5 \text {-in. well }=60 \mathrm{ft} \text {. Sample } \\
\text { collected by using Waterra pump after purging of } 5.2 \mathrm{~L} \text {. Water } \\
\text { brown to tannish in color. }\end{array}$ \\
\hline 9/9/08 & $9: 30$ & CNMW01-W-26673 & MW & MW01 & $54.5-64.5$ & 6123 & $\begin{array}{l}\text { Depth to water }=13.6 \mathrm{ft} \text {. Depth of } 4 \text {-in. well }=64.5 \mathrm{ft} \text {. Sample } \\
\text { collected by using low-flow bladder pump after purging of } 10 \mathrm{~L} \text {. } \\
\text { Water clear. }\end{array}$ \\
\hline 9/9/08 & $10: 52$ & CNSB07R-W-26686 & $\mathrm{CPT} / \mathrm{P}$ & SB07R & $45-60$ & 6123 & $\begin{array}{l}\text { Depth to water }=18.4 \mathrm{ft} \text {. Depth of } 2 \text {-in. well }=60 \mathrm{ft} \text {. Sample } \\
\text { collected by using low-flow bladder pump after purging of } 6 \mathrm{~L} \text {. } \\
\text { Water clear. }\end{array}$ \\
\hline 9/9/08 & $11: 27$ & CNPMP7-W-26695 & MW & PMP7 & $50-60$ & 6123 & $\begin{array}{l}\text { Depth to water }=20 \mathrm{ft} \text {. Depth of } 0.5 \text {-in. well }=60 \mathrm{ft} \text {. Sample } \\
\text { collected by using Waterra pump. Water has light brown tint. }\end{array}$ \\
\hline 9/9/08 & $12: 04$ & CNSB04-W-26684 & CPT/P & SB04 & $51-61$ & 6123 & $\begin{array}{l}\text { Depth to water }=21.9 \mathrm{ft} \text {. Depth of } 1 \text {-in. well }=61 \mathrm{ft} \text {. Sample } \\
\text { collected by using low-flow bladder pump after purging of } \\
3.2 \mathrm{~L} \text {. Water clear. }\end{array}$ \\
\hline 9/9/08 & $12: 33$ & CNPMP4-W-26692 & MW & PMP4 & $48.75-58.75$ & 6123 & $\begin{array}{l}\text { Depth to water }=19.15 \mathrm{ft} \text {. Depth of } 0.5 \text {-in. well }=58.75 \mathrm{ft} \text {. Sample } \\
\text { collected by using Waterra pump after purging of } 5.5 \mathrm{~L} \text {. Water } \\
\text { tannish brown in color. }\end{array}$ \\
\hline 9/9/08 & $12: 54$ & CNMW03-W-26675 & MW & MW03 & $50.5-60.5$ & 6123 & $\begin{array}{l}\text { Depth to water }=20.8 \mathrm{ft} \text {. Depth of } 4 \text {-in. well }=60.5 \mathrm{ft} \text {. Sample } \\
\text { collected by using low-flow bladder pump after purging of } \\
6.3 \mathrm{~L} \text {. Water clear. }\end{array}$ \\
\hline 9/9/08 & $13: 10$ & CNPMP8-W-26696 & MW & PMP8 & $50-60$ & 6123 & $\begin{array}{l}\text { Depth to water }=20.3 \mathrm{ft} \text {. Depth of } 0.5 \text {-in. well }=60 \mathrm{ft} \text {. Sample } \\
\text { collected by using Waterra pump after purging of } 5.5 \mathrm{~L} \text {. Water } \\
\text { tannish in color with odor. }\end{array}$ \\
\hline 9/9/08 & $13: 40$ & CNPMP3-W-26691 & MW & PMP3 & $50-60$ & 6123 & $\begin{array}{l}\text { Depth to water }=21.6 \mathrm{ft} \text {. Depth of } 0.5 \text {-in. well }=60 \mathrm{ft} \text {. Sample } \\
\text { collected by using Waterra pump after purging of } 5.2 \mathrm{~L} \text {. Water } \\
\text { cloudy to grayish tint in color, with odor. }\end{array}$ \\
\hline 9/9/08 & $13: 48$ & CNMW10-W-26682 & MW & MW10 & $30-45$ & 6123 & $\begin{array}{l}\text { Depth to water }=21.1 \mathrm{ft} \text {. Depth of } 2 \text {-in. well }=45 \mathrm{ft} \text {. Sample } \\
\text { collected by using low-flow bladder pump after purging of } 7 \mathrm{~L} \text {. } \\
\text { Water clear. }\end{array}$ \\
\hline
\end{tabular}


TABLE A.1 (Cont.)

\begin{tabular}{|c|c|c|c|c|c|c|c|}
\hline Date & Time & Sample & Type $^{a}$ & Location $^{\mathrm{b}}$ & $\begin{array}{l}\text { Depthc } \\
\text { (ft TOC) }\end{array}$ & $\begin{array}{l}\text { Chain of } \\
\text { Custody }\end{array}$ & Sample Description \\
\hline 9/9/08 & $14: 12$ & CNPMP9-W-26697 & MW & PMP9 & $50-60$ & 6122 & $\begin{array}{l}\text { Depth to water }=16.2 \mathrm{ft} \text {. Depth of } 0.5 \text {-in. well }=60 \mathrm{ft} \text {. Sample } \\
\text { collected by using Waterra pump after purging of } 5.4 \mathrm{~L} \text {. Water } \\
\text { clear to cloudy. }\end{array}$ \\
\hline 9/9/08 & $14: 42$ & CNPMP2-W-26690 & MW & PMP2 & $50-60$ & 6123 & $\begin{array}{l}\text { Depth to water }=20.9 \mathrm{ft} \text {. Depth of } 0.5 \text {-in. well }=60 \mathrm{ft} \text {. Sample } \\
\text { collected by using Waterra pump after purging of } 5.1 \mathrm{~L} \text {. Water } \\
\text { clear to cloudy, with strong odor. }\end{array}$ \\
\hline 9/9/08 & $15: 10$ & CNMW06-W-26678 & MW & MW06 & $46.5-56.5$ & 6123 & $\begin{array}{l}\text { Depth to water }=36.6 \mathrm{ft} \text {. Depth of } 4 \text {-in. well }=56.5 \mathrm{ft} \text {. Sample } \\
\text { collected by using low-flow bladder pump after purging of } \\
6.4 \mathrm{~L} \text {. Water tannish in color. }\end{array}$ \\
\hline 9/9/08 & $15: 12$ & CNPMP1-W-26689 & MW & PMP1 & $50-60$ & 6123 & $\begin{array}{l}\text { Depth to water }=20.9 \mathrm{ft} \text {. Depth of } 0.5 \text {-in. well }=60 \mathrm{ft} \text {. Sample } \\
\text { collected by using Waterra pump after purging of } 5 \mathrm{~L} \text {. Water } \\
\text { brown-tan in color, with odor. }\end{array}$ \\
\hline 9/9/08 & $16: 18$ & CNMW07-W-26679 & MW & MW07 & $45-55$ & 6123 & $\begin{array}{l}\text { Depth to water }=28.2 \mathrm{ft} \text {. Depth of } 2 \text {-in. well }=55 \mathrm{ft} \text {. Sample } \\
\text { collected by using low-flow bladder pump after purging of } 7 \mathrm{~L} \text {. } \\
\text { Water clear. }\end{array}$ \\
\hline 9/9/08 & $16: 59$ & CNSB05-W-26685 & $\mathrm{CPT/P}$ & SB05 & $32-42$ & 6123 & $\begin{array}{l}\text { Depth to water }=10.6 \mathrm{ft} \text {. Depth of } 1 \text {-in. well }=42 \mathrm{ft} \text {. Sample } \\
\text { collected by using Waterra pump after purging of } 15 \mathrm{~L} \text {. Water } \\
\text { clear to cloudy. }\end{array}$ \\
\hline 9/9/08 & $17: 10$ & CNMW04-W-26676 & MW & MW04 & $37.5-47.5$ & 6123 & $\begin{array}{l}\text { Depth to water }=24 \mathrm{ft} \text {. Depth of } 4 \text {-in. well }=47.5 \mathrm{ft} \text {. Sample } \\
\text { collected by using low-flow bladder pump after purging of } \\
6.2 \mathrm{~L} \text {. Water clear. }\end{array}$ \\
\hline 9/10/08 & $9: 22$ & CNMW05-W-26677 & MW & MW05 & $34.5-44.5$ & 6124 & $\begin{array}{l}\text { Depth to water }=10 \mathrm{ft} \text {. Depth of } 4 \text {-in. well }=44.5 \mathrm{ft} \text {. Sample } \\
\text { collected by using low-flow bladder pump after purging of } 5 \mathrm{~L} \text {. } \\
\text { Water clear. }\end{array}$ \\
\hline 9/10/08 & $9: 23$ & CNMW05DUP-W-26698 & MW & MW05 & $34.5-44.5$ & 6124 & Replicate of sample CNMW05-W-26677. \\
\hline 9/10/08 & $10: 22$ & CNPMP5-W-26693 & MW & PMP5 & $50-60$ & 6124 & $\begin{array}{l}\text { Depth to water }=21.4 \mathrm{ft} \text {. Depth of } 1 \text {-in. well }=60 \mathrm{ft} \text {. Sample } \\
\text { collected by using low-flow bladder pump after purging of } 3 \mathrm{~L} \text {. } \\
\text { Water brownish color. }\end{array}$ \\
\hline 9/10/08 & $11: 38$ & CNSB01-W-26683 & $\mathrm{CPT/P}$ & SB01 & $40-50$ & 6124 & $\begin{array}{l}\text { Depth to water }=20.1 \mathrm{ft} \text {. Depth of } 1 \text {-in. well }=50 \mathrm{ft} \text {. Sample } \\
\text { collected by using low-flow bladder pump after purging of } 3 \mathrm{~L} \text {. } \\
\text { Water clear. }\end{array}$ \\
\hline 9/10/08 & $11: 45$ & CNQCIR-W-26700 & RI & QC & - & 6124 & $\begin{array}{l}\text { Rinsate of decontaminated pump purge line after collection of } \\
\text { sample CNSB01-W-26683. }\end{array}$ \\
\hline $9 / 10 / 08$ & $12: 22$ & CNMW09-W-26681 & MW & MW09 & $25-35$ & 6124 & $\begin{array}{l}\text { Depth to water }=2.5 \mathrm{ft} \text {. Depth of } 2 \text {-in. well }=35 \mathrm{ft} \text {. Sample } \\
\text { collected by using low-flow bladder pump after purging of } 6 \mathrm{~L} \text {. }\end{array}$ \\
\hline 9/10/08 & $13: 34$ & CNSB09-W-26688 & $\mathrm{CPT/P}$ & SB09 & $32-42$ & 6124 & $\begin{array}{l}\text { Depth to water }=6.6 \mathrm{ft} \text {. Depth of } 1 \text {-in. well }=42 \mathrm{ft} \text {. Sample } \\
\text { collected by using low-flow bladder pump after purging of } 3 \mathrm{~L} \text {. }\end{array}$ \\
\hline
\end{tabular}


TABLE A.1 (Cont.)

\begin{tabular}{|c|c|c|c|c|c|c|c|}
\hline Date & Time & Sample & Type ${ }^{a}$ & Location $^{b}$ & $\begin{array}{c}\text { Depthc } \\
\text { (ft TOC) }\end{array}$ & $\begin{array}{l}\text { Chain of } \\
\text { Custody }\end{array}$ & Sample Description \\
\hline 9/10/08 & $13: 45$ & CNQCIR-W-26701 & $\mathrm{RI}$ & $\mathrm{QC}$ & - & 6124 & $\begin{array}{l}\text { Rinsate of decontaminated pump purge line after collection of } \\
\text { sample CNMW09-W-26681. }\end{array}$ \\
\hline 9/10/08 & $14: 28$ & CNMW08-W-26680 & MW & MW08 & $38-53$ & 6124 & $\begin{array}{l}\text { Depth to water }=18.6 \mathrm{ft} \text {. Depth of } 2 \text {-in. well }=53 \mathrm{ft} \text {. Sample } \\
\text { collected by using low-flow bladder pump after purging of } \\
6.5 \mathrm{~L} \text {. Water clear. }\end{array}$ \\
\hline 9/10/08 & $15: 00$ & CNQCTB-W-26702 & TB & $\mathrm{QC}$ & - & $\begin{array}{l}6122 \\
6123 \\
6124 \\
6134\end{array}$ & $\begin{array}{l}\text { Trip blank sent to the AGEM Laboratory for organic analyses with } \\
\text { water samples listed on chain-of-custody forms (COCs) } 6122 \text {, } \\
6123 \text {, and } 6124, \text { as well as to TestAmerica for verification } \\
\text { organic analysis with samples listed on COC } 6134 \text {. }\end{array}$ \\
\hline
\end{tabular}

a Sample types: CPT/P, cone penetrometer piezometer; RI, rinsate; MW, monitoring well; TB, trip blank.

b PMP wells were installed in September 2008 in the pilot test treatment zone. Details for these wells were reported separately (Argonne 2009).

c Depths were measured from the top of the casing (TOC). 


\section{Appendix B:}

\section{Data Summary for Verification VOCs Analyses} by TestAmerica Laboratories, Inc. 


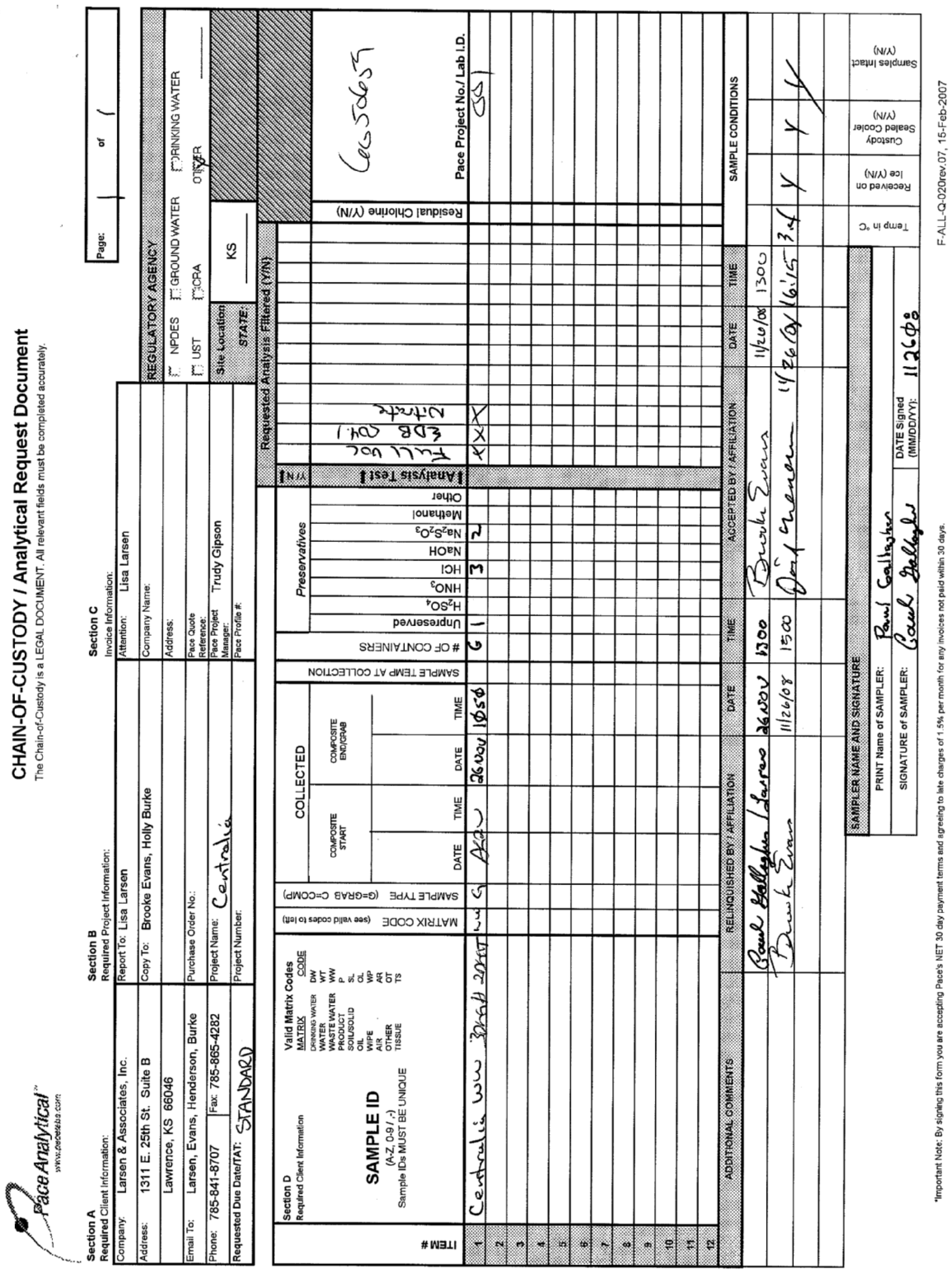




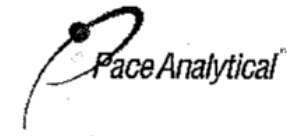

Courier: $\square$ Fed Ex $\square$ UPS $\square$ uSPS
Tracking \#:
Custody Seal on Cooler/Box Present:
Packing Material: $\square$ Bubble Wrap
Thermometer Used
Cooler Temperature
Temp should be above freezing to $6^{\circ} \mathrm{C}$

Chain of Custody Present:

Chain of Custody Filled Out:

Chain of Custody Relinquished:

Sampler Name \& Signature on COC:

Samples Arrived within Hold Time:

Short Hold Time Analysis $(<72 \mathrm{hr})$ :

Rush Turn Around Time Requested:

Sufficient Volume:

Correct Containers Used:

-Pace Containers Used:

Containers Intact:

Filtered volume received for Dissolved tests

Sample Labels match COC:

-Includes date/time/ID/Analysis Matrix:

All containers needing preservation have been checked.

All containers needing preservation are found to be in compliance with EPA recommendation.

exceptions: VØA, coliform, TOC, O\&G, WI-DRO (water)

Samples checked for dechlorination:

Headspace in VOA Vials $(>6 \mathrm{~mm})$ :

Trip Blank Present:

Trip Blank Custody Seals Present

Pace Trip Blank Lot \# (if purchased):

\section{Sample Condition Upon Receipt}

Project \# Cas0655

\begin{tabular}{|c|}
\hline Optional \\
\hline Proj. Due Date: $/ 2 / / 3$ \\
Proj. Name: \\
Ceobr5 \\
\hline
\end{tabular}
Kares

le Bags $\square$ None $\square$ Other

$$
\text { . }
$$
Type of Ice: We Blue None

$\square$ Samples on ice, cooling process has begun Biological Tissue is Frozen: Yes No Date and Initials of person examining Comments: Date and Initials of person examining
contents: $\quad$ \&s $1 / 2 a / a r$

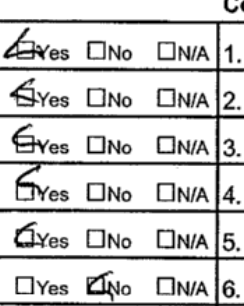
口Yes GNo DN/A 7. Gives पNo पN/A 8. CTres $\square$ No $\square$ N/A 9 . EYes पno पN/A

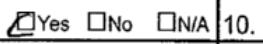

\begin{tabular}{ll}
\hline $\begin{array}{l}\text { Client Notification/ Resolution: } \\
\text { Person Contacted: }\end{array}$ & Field Data Required? \\
Comments/ Resolution: & Date/Time:
\end{tabular}

\begin{tabular}{llll}
\hline & & \\
\hline & & & \\
\hline & Sroject Manager Review: 11.28 .08 & Date:
\end{tabular}

Note: Whenever there is a discrepancy affecting North Carolina compliance samples, a copy of this form will be sent to the North Carolina DEHNR Certification Office ( i.e out of hold, incorrect preservative, out of temp, incorrect containers) 
Lisa Larsen

Larsen \& Associates, Inc.

1311 E. 25th St.

Suite B

Lawrence, KS 66046

RE: Project: Centralia

Pace Project No.: 6050659

Dear Lisa Larsen:

Enclosed are the analytical results for sample(s) received by the laboratory on November 26, 2008. The results relate only to the samples included in this report. Results reported herein conform to the most current NELAC standards, where applicable, unless otherwise narrated in the body of the report.

If you have any questions concerning this report, please feel free to contact me.

Sincerely,

$$
\text { sudy sijoson }
$$

Trudy Gipson

trudy.gipson@pacelabs.com

Project Manager

\section{Enclosures}

cc: Holly Burke, Larsen \& Associates, Inc Brooke Evans, Larsen \& Associates, Inc. 


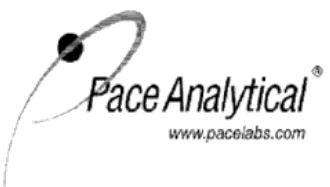

Pace Analytical Services, Inc.

9608 Loiret Blvd.

Lenexa, KS 66219

(913)599-5665

\section{CERTIFICATIONS}

Project: Centralia

Pace Project No.: 6050659

Kansas Certification IDs

Utah Certification Number: 9135995665

lowa Certification Number: 118

Texas Certification Number: T104704407-08-TX

Oklahoma Certification Number: 9205/9935

Illinois Certification Number: 001191

Nevada Certification Number: KSO00212008A

Arkansas Certification Number: 05-008-0

Louisiana Certification Number: 03055

Kansas/NELAP Certification Number: E-10116

\footnotetext{
This report shall not be reproduced, except in full,
}

without the written consent of Pace Analytical Services, Inc.

$$
\text { nelac }
$$




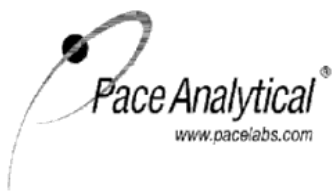

(913)599-5665

SAMPLE SUMMARY

\begin{tabular}{|c|c|c|c|c|}
\hline Project: & Centralia & & & \\
\hline Pace Project No.: & 6050659 & & & \\
\hline Lab ID & Sample ID & Matrix & Date Collected & Date Received \\
\hline 6050659001 & CENTRALIA WW & Water & $11 / 26 / 08$ 10:50 & $11 / 26 / 08$ 16:15 \\
\hline
\end{tabular}

This report shall not be reproduced, except in full, 


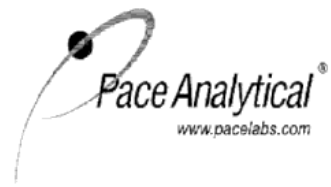

SAMPLE ANALYTE COUNT

$\begin{array}{ll}\text { Project: } & \text { Centralia } \\ \text { Pace Project No.: } & 6050659\end{array}$

\begin{tabular}{|c|c|c|c|c|}
\hline Lab ID & Sample ID & Method & Analysts & $\begin{array}{l}\text { Analytes } \\
\text { Reported }\end{array}$ \\
\hline \multirow[t]{3}{*}{6050659001} & CENTRALIA WW & EPA 300.0 & AJM & 1 \\
\hline & & EPA $5030 B / 8260$ & AJA & 71 \\
\hline & & EPA 504.1 & SRM & 1 \\
\hline
\end{tabular}




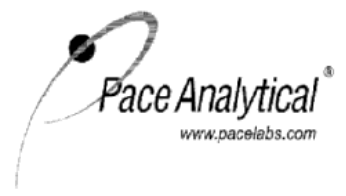

\section{ANALYTICAL RESULTS}

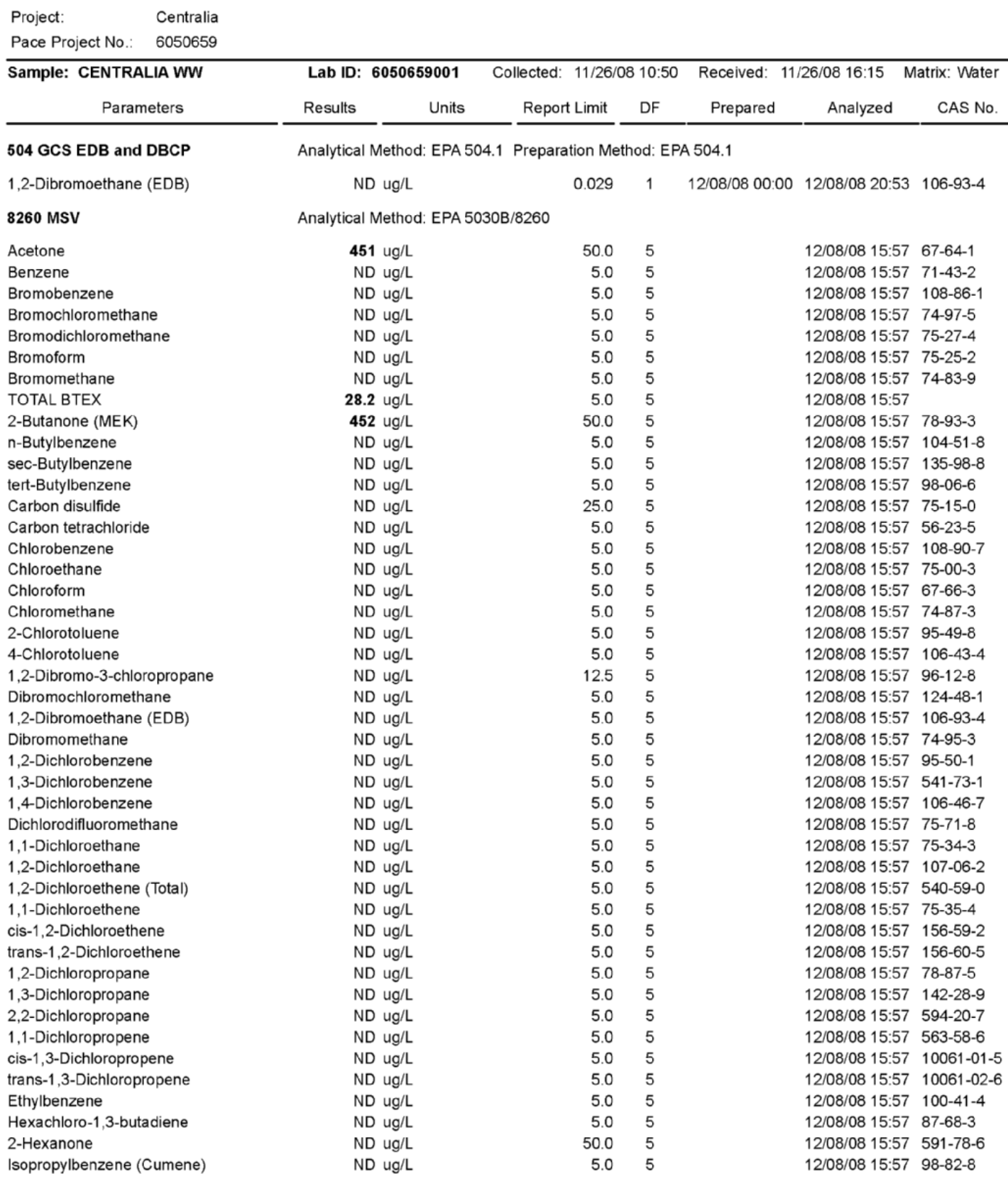

REPORT OF LABORATORY ANALYSIS 


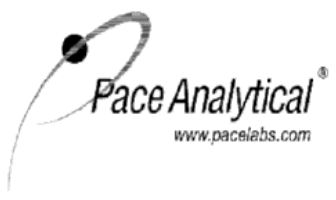

ANALYTICAL RESULTS

Project: Centralia

Pace Project No.: 6050659

\begin{tabular}{|c|c|c|c|c|c|c|c|}
\hline \multirow{2}{*}{$\begin{array}{c}\text { Sample: CENTRALIA WW } \\
\text { Parameters }\end{array}$} & Lab ID: 6050659001 & \multicolumn{2}{|c|}{ Collected: $11 / 26 / 08$ 10:50 } & \multicolumn{2}{|c|}{ Received: $11 / 26 / 08$ 16:15 } & Matrix: Water & \multirow[b]{2}{*}{ Qual } \\
\hline & Results & Report Limit & DF & Prepared & Analyzed & CAS No. & \\
\hline 8260 MSV & Analytical Method: EPA 5 & $30 \mathrm{~B} / 8260$ & & & & & \\
\hline p-lsopropyltoluene & ND ug/L & 5.0 & 5 & & $12 / 08 / 0815: 57$ & $99-87-6$ & \\
\hline Methylene chloride & ND ug/L & 5.0 & 5 & & $12 / 08 / 0815: 57$ & $75-09-2$ & \\
\hline 4-Methyl-2-pentanone (MIBK) & ND ug/L & 50.0 & 5 & & $12 / 08 / 0815: 57$ & $108-10-1$ & \\
\hline Methyl-tert-butyl ether & ND ug/L & 5.0 & 5 & & $12 / 08 / 0815: 57$ & $1634-04-4$ & \\
\hline Naphthalene & ND ug/L & 50.0 & 5 & & $12 / 08 / 0815: 57$ & $91-20-3$ & \\
\hline n-Propylbenzene & ND ug/L & 5.0 & 5 & & $12 / 08 / 0815: 57$ & $103-65-1$ & \\
\hline Styrene & ND ug/L & 5.0 & 5 & & $12 / 08 / 0815: 57$ & $100-42-5$ & \\
\hline 1,1,1,2-Tetrachloroethane & ND ug/L & 5.0 & 5 & & $12 / 08 / 0815: 57$ & $630-20-6$ & \\
\hline 1,1,2,2-Tetrachloroethane & ND ug/L & 5.0 & 5 & & $12 / 08 / 0815: 57$ & $79-34-5$ & \\
\hline Tetrachloroethene & ND ug/L & 5.0 & 5 & & $12 / 08 / 0815: 57$ & $127-18-4$ & \\
\hline Toluene & $15.2 \mathrm{ug} / \mathrm{L}$ & 5.0 & 5 & & $12 / 08 / 0815: 57$ & $108-88-3$ & \\
\hline 1,2,3-Trichlorobenzene & ND ug/L & 5.0 & 5 & & $12 / 08 / 0815: 57$ & $87-61-6$ & \\
\hline 1,2,4-Trichlorobenzene & ND ug/L & 5.0 & 5 & & $12 / 08 / 0815: 57$ & $120-82-1$ & \\
\hline 1,1,1-Trichloroethane & ND ug/L & 5.0 & 5 & & $12 / 08 / 0815: 57$ & $71-55-6$ & \\
\hline 1,1,2-Trichloroethane & ND ug/L & 5.0 & 5 & & $12 / 08 / 0815: 57$ & $79-00-5$ & \\
\hline Trichloroethene & ND ug/L & 5.0 & 5 & & $12 / 08 / 0815: 57$ & $79-01-6$ & \\
\hline Trichlorofluoromethane & ND ug/L & 5.0 & 5 & & $12 / 08 / 0815: 57$ & $75-69-4$ & \\
\hline 1,2,3-Trichloropropane & ND ug/L & 12.5 & 5 & & $12 / 08 / 0815: 57$ & $96-18-4$ & \\
\hline 1,2,4-Trimethylbenzene & ND ug/L & 5.0 & 5 & & $12 / 08 / 0815: 57$ & $95-63-6$ & \\
\hline 1,3,5-Trimethylbenzene & ND ug/L & 5.0 & 5 & & $12 / 08 / 0815: 57$ & $108-67-8$ & \\
\hline Vinyl chloride & ND ug/L & 5.0 & 5 & & $12 / 08 / 0815: 57$ & $75-01-4$ & \\
\hline Xylene (Total) & $16.1 \mathrm{ug} / \mathrm{L}$ & 15.0 & 5 & & $12 / 08 / 0815: 57$ & $1330-20-7$ & \\
\hline 4-Bromofluorobenzene (S) & $105 \%$ & $85-119$ & 5 & & $12 / 08 / 0815: 57$ & $460-00-4$ & \\
\hline Dibromofluoromethane (S) & $104 \%$ & $85-114$ & 5 & & $12 / 08 / 0815: 57$ & $1868-53-7$ & \\
\hline 1,2-Dichloroethane-d4 (S) & $107 \%$ & $81-118$ & 5 & & $12 / 08 / 0815: 57$ & $17060-07-0$ & \\
\hline Toluene-d8 (S) & $99 \%$ & $82-114$ & 5 & & $12 / 08 / 0815: 57$ & $2037-26-5$ & \\
\hline Preservation $\mathrm{pH}$ & 1.0 & 0.10 & 5 & & $12 / 08 / 0815: 57$ & & \\
\hline 300.0 IC Anions & Analytical Method: EPA 3 & & & & & & \\
\hline Nitrate as $\mathrm{N}$ & $\mathrm{ND} \mathrm{mg} / \mathrm{L}$ & 0.10 & 1 & & $11 / 28 / 0809: 57$ & $14797-55-8$ & \\
\hline
\end{tabular}




\section{QUALITY CONTROL DATA}

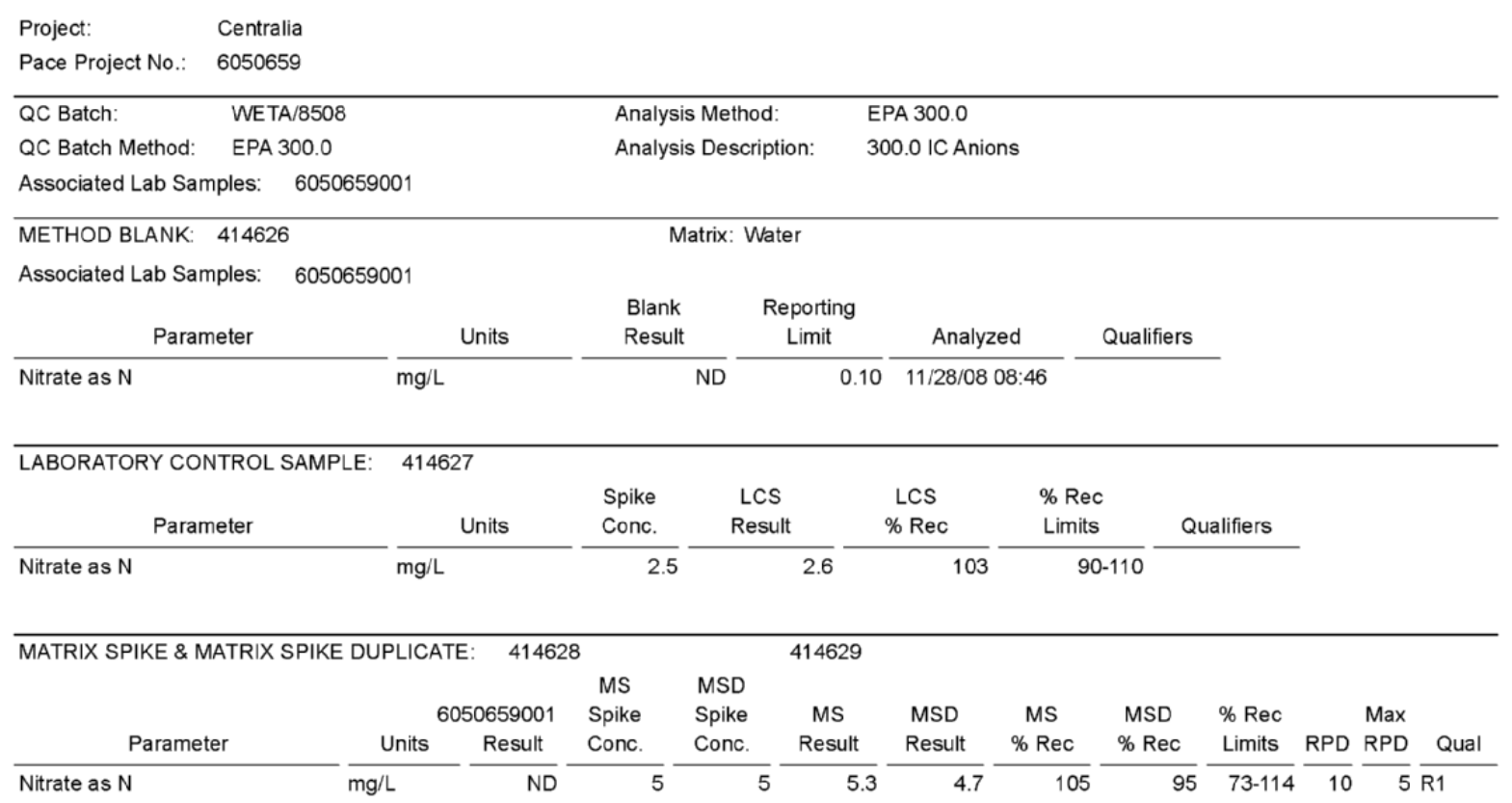


QUALITY CONTROL DATA

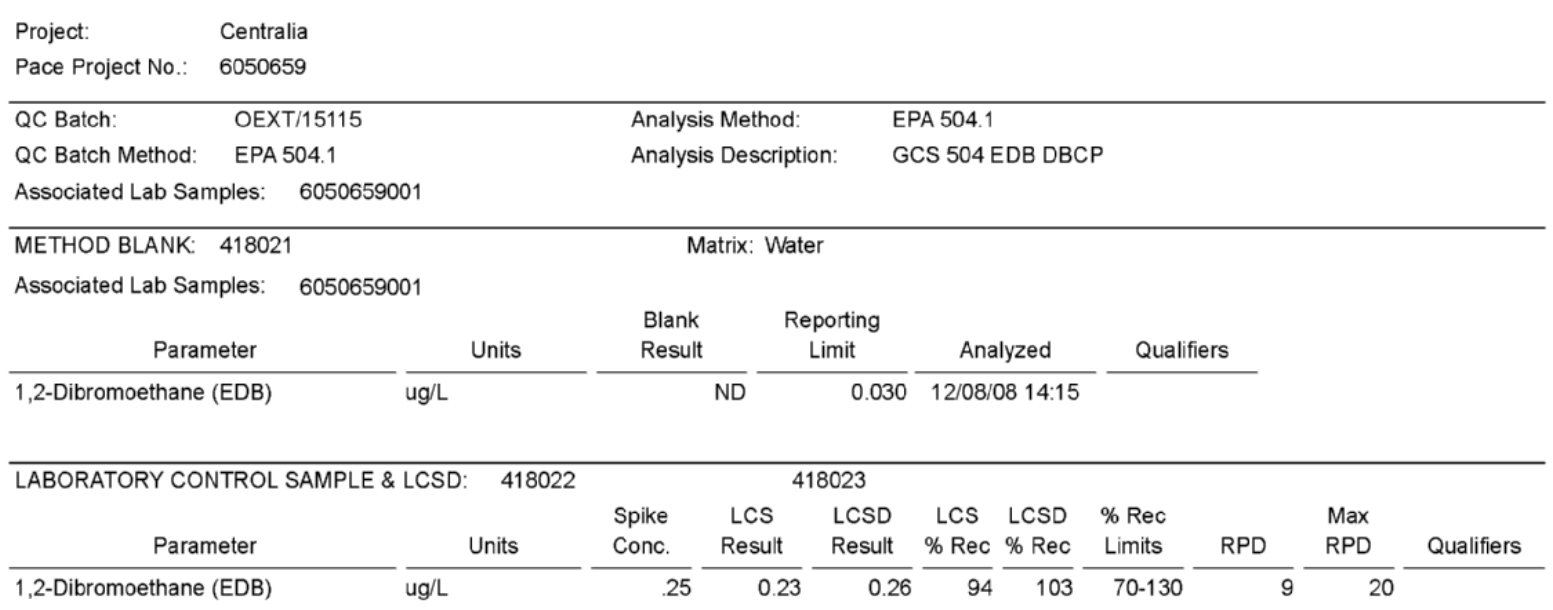

This report shall not be reproduced, except in full, without the written consent of Pace Analytical Services, Inc.. 


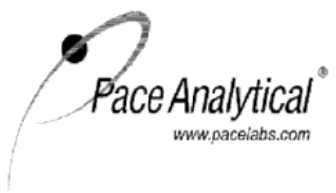

Pace Analytical Services, Inc.

9608 Loiret Blvd

Lenexa, KS 66219

(913)599-5665

\section{QUALITY CONTROL DATA}

$\begin{array}{ll}\text { Project: } & \text { Centralia } \\ \text { Pace Project No.: } & 6050659\end{array}$

\begin{tabular}{llll}
\hline QC Batch: & MSV/18481 & Analysis Method: & EPA 5030B/8260 \\
QC Batch Method: & EPA 5030B/8260 & Analysis Description: & 8260 MSV Water 10 mL Purge \\
Associated Lab Samples: $\quad 6050659001$ & & \\
\hline
\end{tabular}

METHOD BLANK: 418050

Matrix: Water

Associated Lab Samples: 6050659001

$\begin{array}{llll} & \text { Blank Reporting }\end{array}$

Units Limit

ug/l

$u g / L$

1,1,2,2-Tetrachloroethane ug/L

1,1,2-Trichloroethane ug/L

1,1-Dichloroethane ug/L

1,1-Dichloroethene ug/L

1,1-Dichloropropene ug/L

1,2,3-Trichlorobenzene ug/L

1,2,3-Trichloropropane ug/L

1,2,4-Trichlorobenzene ug/L

1,2,4-Trimethylbenzene ug/L

1,2-Dibromo-3-chloropropane ug/L

1,2-Dibromoethane (EDB) ug/L

1,2-Dichlorobenzene ug/L

1,2-Dichloroethane ug/L

1,2-Dichloroethene (Total) ug/L

1,2-Dichloropropane ug/L

1,3,5-Trimethylbenzene ug/L

1,3-Dichlorobenzene ug/L

1,3-Dichloropropane ug/L

1,4-Dichlorobenzene ug/L

2,2-Dichloropropane ug/L

2-Butanone (MEK) ug/L

2-Chlorotoluene ug/L

2-Hexanone ug/L

4-Chlorotoluene ug/L

4-Methyl-2-pentanone (MIBK) ug/L

Acetone

Benzene

Bromobenzene

Bromochloromethane

Bromodichloromethane

Bromoform

Bromomethane

Carbon disulfide

Carbon tetrachloride

Chlorobenzene

Chloroethane

Chloroform

Chloromethane

cis-1,2-Dichloroethene

cis-1,3-Dichloropropene

Dibromochloromethane

Date: 12/10/2008 03:08 PM 


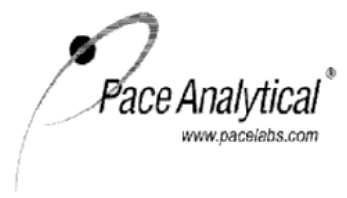

Pace Analytical Services, Inc. 9608 Loiret Blvd Lenexa, KS 66219 (913)599-5665

QUALITY CONTROL DATA

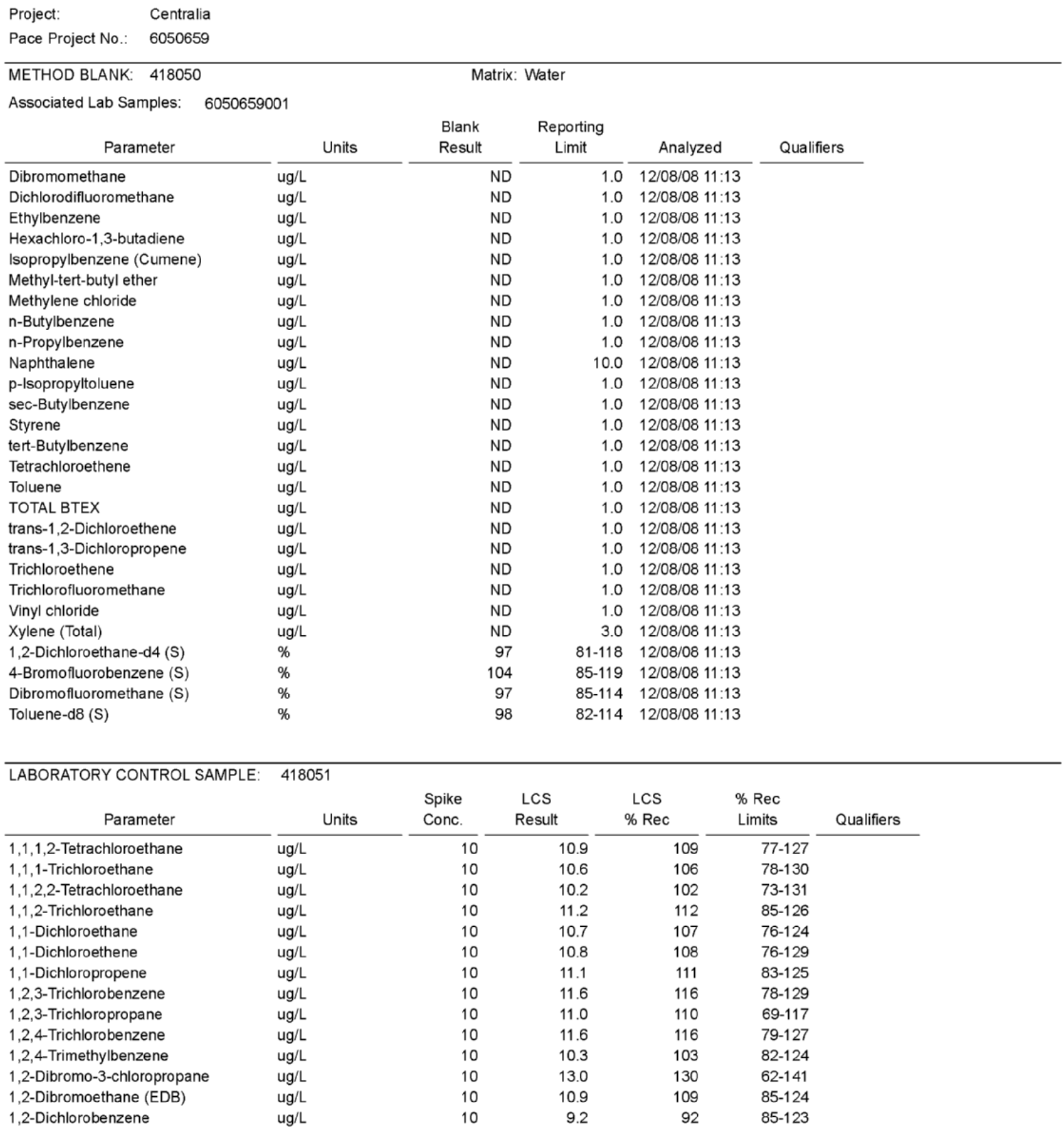




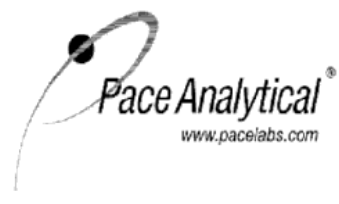

Pace Analytical Services, Inc. 9608 Loiret Blvd. Lenexa, KS 66219 (913)599-5665

QUALITY CONTROL DATA

$\begin{array}{ll}\text { Project: } & \text { Centralia } \\ \text { Pace Project No.: } & 6050659\end{array}$

\begin{tabular}{|c|c|c|c|c|c|c|}
\hline \multirow{2}{*}{$\begin{array}{c}\text { LABORATORY CONTROL SAMPLE } \\
\text { Parameter }\end{array}$} & \multicolumn{6}{|l|}{418051} \\
\hline & Units & $\begin{array}{l}\text { Spike } \\
\text { Conc. }\end{array}$ & $\begin{array}{l}\text { LCS } \\
\text { Result }\end{array}$ & $\begin{array}{c}\text { LCS } \\
\% \operatorname{Rec}\end{array}$ & $\begin{array}{l}\% \text { Rec } \\
\text { Limits }\end{array}$ & Qualifiers \\
\hline 1,2-Dichloroethane & $\mathrm{ug} / \mathrm{L}$ & 10 & 10.3 & 103 & $77-129$ & \\
\hline 1,2-Dichloroethene (Total) & $\mathrm{ug} / \mathrm{L}$ & 20 & 21.0 & 105 & $81-127$ & \\
\hline 1,2-Dichloropropane & $\mathrm{ug} / \mathrm{L}$ & 10 & 10.7 & 107 & $82-121$ & \\
\hline 1,3,5-Trimethylbenzene & $\mathrm{ug} / \mathrm{L}$ & 10 & 9.9 & 99 & $85-122$ & \\
\hline 1,3-Dichlorobenzene & ug/L & 10 & 9.5 & 95 & $84-121$ & \\
\hline 1,3-Dichloropropane & $\mathrm{ug} / \mathrm{L}$ & 10 & 10.1 & 101 & $86-121$ & \\
\hline 1,4-Dichlorobenzene & ug/L & 10 & 9.5 & 95 & $83-121$ & \\
\hline 2,2-Dichloropropane & $\mathrm{ug} / \mathrm{L}$ & 10 & 11.6 & 116 & $47-154$ & \\
\hline 2-Butanone (MEK) & ug/L & 25 & 25.1 & 101 & $64-126$ & \\
\hline 2-Chlorotoluene & $\mathrm{ug} / \mathrm{L}$ & 10 & 9.2 & 92 & $83-125$ & \\
\hline 2-Hexanone & $\mathrm{ug} / \mathrm{L}$ & 25 & 28.9 & 116 & $65-128$ & \\
\hline 4-Chlorotoluene & ug/L & 10 & 9.6 & 96 & $84-121$ & \\
\hline 4-Methyl-2-pentanone (MIBK) & $\mathrm{ug} / \mathrm{L}$ & 25 & 26.5 & 106 & $64-121$ & \\
\hline Acetone & ug/L & 25 & 25.6 & 102 & $52-139$ & \\
\hline Benzene & $\mathrm{ug} / \mathrm{L}$ & 10 & 10.6 & 106 & $87-117$ & \\
\hline Bromobenzene & $\mathrm{ug} / \mathrm{L}$ & 10 & 9.7 & 97 & 83-126 & \\
\hline Bromochloromethane & $\mathrm{ug} / \mathrm{L}$ & 10 & 9.6 & 96 & $82-129$ & \\
\hline Bromodichloromethane & $\mathrm{ug} / \mathrm{L}$ & 10 & 10.6 & 106 & $75-127$ & \\
\hline Bromoform & $\mathrm{ug} / \mathrm{L}$ & 10 & 11.0 & 110 & $64-133$ & \\
\hline Bromomethane & $\mathrm{ug} / \mathrm{L}$ & 10 & 10 & 100 & $21-188$ & \\
\hline Carbon disulfide & ug/L & 10 & 11.8 & 118 & $53-120$ & \\
\hline Carbon tetrachloride & $\mathrm{ug} / \mathrm{L}$ & 10 & 12.1 & 121 & $76-131$ & \\
\hline Chlorobenzene & $\mathrm{ug} / \mathrm{L}$ & 10 & 9.8 & 98 & $85-120$ & \\
\hline Chloroethane & $\mathrm{ug} / \mathrm{L}$ & 10 & 8.8 & 88 & $69-126$ & \\
\hline Chloroform & $\mathrm{ug} / \mathrm{L}$ & 10 & 10.3 & 103 & $79-126$ & \\
\hline Chloromethane & $\mathrm{ug} / \mathrm{L}$ & 10 & 6.7 & 67 & $44-118$ & \\
\hline cis-1,2-Dichloroethene & $\mathrm{ug} / \mathrm{L}$ & 10 & 10.4 & 104 & $79-128$ & \\
\hline cis-1,3-Dichloropropene & ug/L & 10 & 11.1 & 111 & $76-122$ & \\
\hline Dibromochloromethane & $\mathrm{ug} / \mathrm{L}$ & 10 & 11.3 & 113 & $74-121$ & \\
\hline Dibromomethane & $\mathrm{ug} / \mathrm{L}$ & 10 & 10.6 & 106 & $75-130$ & \\
\hline Dichlorodifluoromethane & $\mathrm{ug} / \mathrm{L}$ & 10 & 4.7 & 47 & $12-132$ & \\
\hline Ethylbenzene & $\mathrm{ug} / \mathrm{L}$ & 10 & 10.4 & 104 & $84-123$ & \\
\hline Hexachloro-1,3-butadiene & $\mathrm{ug} / \mathrm{L}$ & 10 & 11.9 & 119 & $71-144$ & \\
\hline Isopropylbenzene (Cumene) & $\mathrm{ug} / \mathrm{L}$ & 10 & 8.8 & 88 & $72-107$ & \\
\hline Methyl-tert-butyl ether & $\mathrm{ug} / \mathrm{L}$ & 10 & 9.9 & 99 & $69-115$ & \\
\hline Methylene chloride & $\mathrm{ug} / \mathrm{L}$ & 10 & 10.2 & 102 & $74-132$ & \\
\hline n-Butylbenzene & ug/L & 10 & 9.9 & 99 & $80-126$ & \\
\hline n-Propylbenzene & $\mathrm{ug} / \mathrm{L}$ & 10 & 9.8 & 98 & 83-123 & \\
\hline Naphthalene & $\mathrm{ug} / \mathrm{L}$ & 10 & $8.7 \mathrm{~J}$ & 87 & $61-150$ & \\
\hline p-Isopropyltoluene & ug/L & 10 & 9.9 & 99 & $82-118$ & \\
\hline sec-Butylbenzene & $\mathrm{ug} / \mathrm{L}$ & 10 & 10.1 & 101 & $84-121$ & \\
\hline Styrene & $\mathrm{ug} / \mathrm{L}$ & 10 & 9.9 & 99 & $84-128$ & \\
\hline tert-Butylbenzene & $\mathrm{ug} / \mathrm{L}$ & 10 & 9.6 & 96 & $83-124$ & \\
\hline Tetrachloroethene & $\mathrm{ug} / \mathrm{L}$ & 10 & 9.8 & 98 & $83-126$ & \\
\hline Toluene & $\mathrm{ug} / \mathrm{L}$ & 10 & 10.5 & 105 & $81-124$ & \\
\hline TOTAL BTEX & $\mathrm{ug} / \mathrm{L}$ & & 61.8 & & & \\
\hline trans-1,2-Dichloroethene & ug/L & 10 & 10.6 & 106 & $80-130$ & \\
\hline trans-1,3-Dichloropropene & ug/L & 10 & 10.2 & 102 & $75-122$ & \\
\hline
\end{tabular}

Date: 12/10/2008 03:08 PM 
Pace Analytical Services, Inc. 9608 Loiret Blvd. Lenexa, KS 66219 (913)599-5665

QUALITY CONTROL DATA

\begin{tabular}{|c|c|c|c|c|c|c|c|}
\hline Project: & Centralia & & & & & & \\
\hline Project No.: & 50659 & & & & & & \\
\hline LABORATORY CC & NTROL SAMPLE: & 418051 & & & & & \\
\hline & & & Spike & LCS & LCS & $\% \operatorname{Rec}$ & \\
\hline Para & meter & Units & Conc. & Result & $\% \operatorname{Rec}$ & Limits & Qualifiers \\
\hline Trichloroethene & & $\mathrm{ug} / \mathrm{L}$ & 10 & 10.6 & 106 & $80-130$ & \\
\hline Trichlorofluoromet| & Iane & $\mathrm{ug} / \mathrm{L}$ & 10 & 9.7 & 97 & $65-113$ & \\
\hline Vinyl chloride & & ug/L & 10 & 9.7 & 97 & $59-124$ & \\
\hline Xylene (Total) & & ug/L & 30 & 30.3 & 101 & $83-125$ & \\
\hline 1,2-Dichloroethane & $-d 4(S)$ & $\%$ & & & 106 & $81-118$ & \\
\hline 4-Bromofluoroben & ene (S) & $\%$ & & & 102 & $85-119$ & \\
\hline Dibromofluoromett & lane $(\mathrm{S})$ & $\%$ & & & 101 & $85-114$ & \\
\hline Toluene-d8 (S) & & $\%$ & & & 101 & $82-114$ & \\
\hline
\end{tabular}




\section{QUALIFIERS}

\begin{tabular}{ll} 
Project: & Centralia \\
Pace Project No.: & 6050659 \\
\hline
\end{tabular}

DEFINITIONS

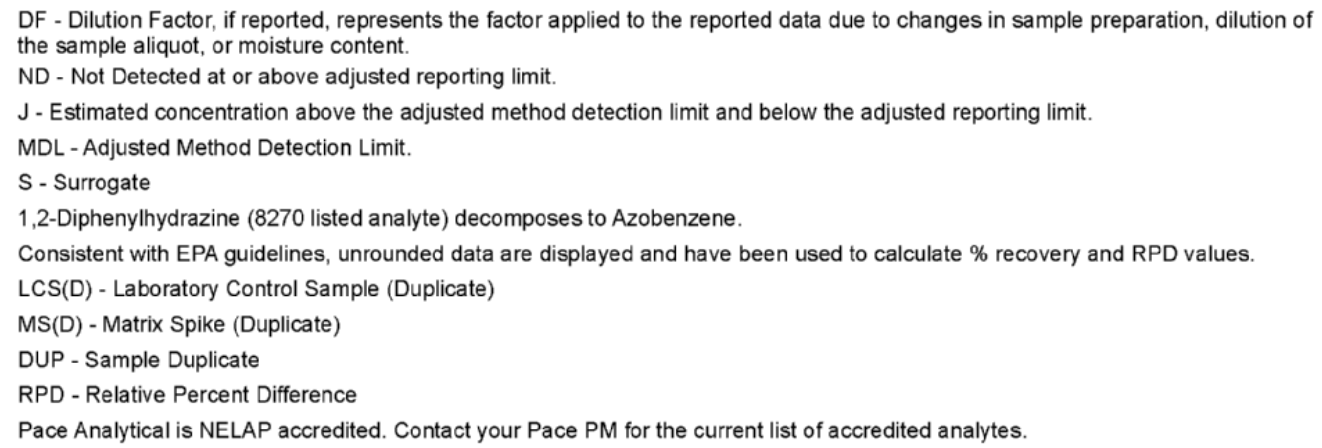

DF - Dilution Factor, if reported, represents the factor applied to the reported data due to changes in sample preparation, dilution of the sample aliquot, or moisture content.

ND - Not Detected at or above adjusted reporting limit.

$\mathrm{J}$ - Estimated concentration above the adjusted method detection limit and below the adjusted reporting limit. MDL - Adjusted Method Detection Limit.

S - Surrogate

1,2-Diphenylhydrazine ( 8270 listed analyte) decomposes to Azobenzene

Consistent with EPA guidelines, unrounded data are displayed and have been used to calculate \% recovery and RPD values. LCS(D) - Laboratory Control Sample (Duplicate)

MS(D) - Matrix Spike (Duplicate)

DUP - Sample Duplicate

RPD - Relative Percent Difference

Pace Analytical is NELAP accredited. Contact your Pace PM for the current list of accredited analytes.

\section{BATCH QUALIFIERS}

Batch: MSV/18481

[1] A matrix spike/matrix spike duplicate was not performed for this batch due to insufficient sample volume

\section{ANALYTE QUALIFIERS}

RPD value was outside control limits. 
QUALITY CONTROL DATA CROSS REFERENCE TABLE

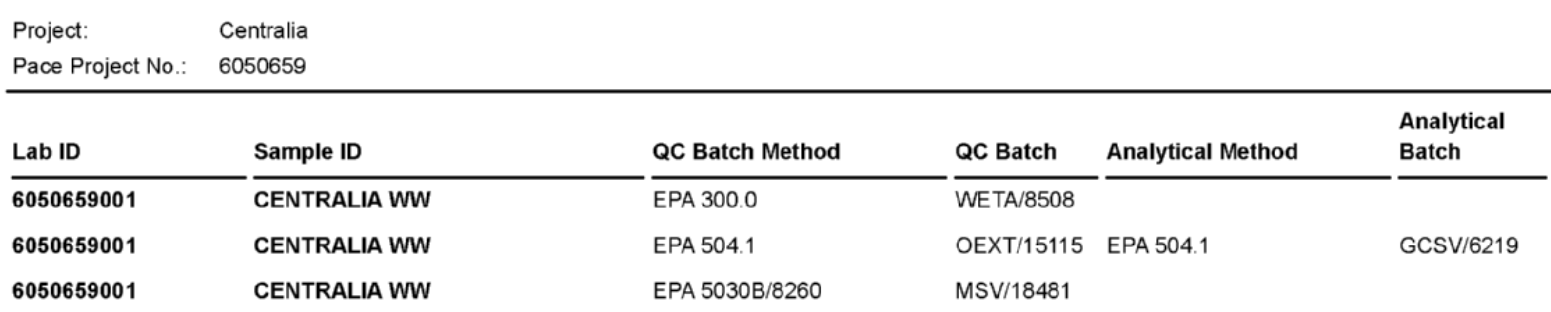


Argonne

Environmental Science Division

Argonne National Laboratory

9700 South Cass Avenue, Bldg. 203

Argonne, IL 60439-4843

www.anl.gov 